\title{
The relationship between Class I and Class II methanol masers at high angular resolution
}

\author{
T. P. McCarthy, ${ }^{1,2 \star}$ S. P. Ellingsen, ${ }^{1}$ M. A. Voronkov ${ }^{2}$ and G. Cimò ${ }^{3,4}$ \\ ${ }^{1}$ School of Natural Sciences, University of Tasmania, Private Bag 37, Hobart, Tasmania 7001, Australia \\ ${ }^{2}$ Australia Telescope National Facility, CSIRO, PO Box 76, Epping, NSW 1710, Australia \\ ${ }^{3}$ Joint Institute for VLBI ERIC (JIVE), Postbus 2, NL-7990AA, Dwingeloo, the Netherlands \\ ${ }^{4}$ Netherlands Institute for Radio Astronomy (ASTRON), Postbus 2, NL-7990AA, Dwingeloo, the Netherlands
}

Accepted 2018 March 1. Received 2018 February 27; in original form 2017 November 10

\begin{abstract}
We have used the Australia Telescope Compact Array (ATCA) to make the first high-resolution observations of a large sample of class I methanol masers in the $95-\mathrm{GHz}\left(8_{0}-7_{1} \mathrm{~A}^{+}\right)$transition. The target sources consist of a statistically complete sample of $6.7-\mathrm{GHz}$ class II methanol masers with an associated $95-\mathrm{GHz}$ class I methanol maser, enabling a detailed study of the relationship between the two methanol maser classes at arcsecond angular resolution. These sources have been previously observed at high resolution in the 36- and 44-GHz transitions, allowing comparison between all three class I maser transitions. In total, 172 95-GHz maser components were detected across the 32 target sources. We find that at high resolution, when considering matched maser components, a 3:1 flux density ratio is observed between the 95- and 44-GHz components, consistent with a number of previous lower angular resolution studies. The $95-\mathrm{GHz}$ maser components appear to be preferentially located closer to the driving sources and this may indicate that this transition is more strongly inverted nearby to background continuum sources. We do not observe an elevated association rate between $95-\mathrm{GHz}$ maser emission and more evolved sources, as indicated by the presence of $12.2-\mathrm{GHz}$ class II masers. We find that in the majority of cases where both class I and class II methanol emission is observed, some component of the class I emission is associated with a likely outflow candidate.
\end{abstract}

Key words: masers-radio lines: ISM.

\section{INTRODUCTION}

Interstellar masers are one of the most readily observable signposts of young high-mass star formation regions as strong emission is commonly observed towards them from a range of molecular species and transitions. The most commonly observed maser species in star formation regions are from the $\mathrm{OH}$, water, and methanol molecules, with the latter having the richest centimetre wavelength spectrum of the group. Methanol is an asymmetric top molecule with hindered internal rotation around the $\mathrm{C}-\mathrm{O}$ bond and it is these characteristics which produce a large number of near-degenerate rotational energy levels. Methanol maser transitions are empirically divided into two classes, known as class I and class II. The class II masers are the better studied methanol masers, with more than 1200 sources having been observed in the $6.7-\mathrm{GHz}$ transition throughout the Milky Way (e.g. Caswell et al. 2010, 2011; Green et al. 2010, 2012, 2017; Breen et al. 2015) and more than 20 different

^E-mail: tiegem@utas.edu.au transitions having been detected (see Ellingsen et al. 2012, and references therein). Class II transitions are closely associated with main-line $\mathrm{OH}$ masers, water masers and are found in compact clusters (a typical linear scale of less than $0.03 \mathrm{pc}$; Caswell 1997) close to the infrared sources which mark the location of a young, highmass star. Class I methanol masers have historically been harder to study as the strongest common transitions are at frequencies of 36- and 44-GHz, which are accessible to less telescopes and furthermore, until recently there was no reliable method for targeting searches for these masers. The class I methanol masers are often observed to be distributed over scales of $0.1-1 \mathrm{pc}$ and are further offset from high-mass stars than class II masers (e.g. Kurtz, Hofner \& Álvarez 2004; Voronkov et al. 2006). The empirically observed difference between the two classes of methanol masers is known to be caused by different pumping mechanisms (Cragg et al. 1992; Voronkov et al. 2005), with the class I methanol masers being collisionally pumped, while the class II masers are radiatively pumped (Sobolev, Cragg \& Godfrey 1997; Cragg, Sobolev \& Godfrey 2005). Class I masers are typically associated with the interface regions of molecular outflows (e.g Kurtz et al. 2004; Cyganowski et al. 2012; 
Voronkov et al. 2006, 2014). However, these masers may also be associated with shocks resulting from expansion of $\mathrm{H}$ II regions, EGOs, or cloud-cloud interactions (Cyganowski et al. 2009, 2012; Sjouwerman et al. 2010; Voronkov et al. 2010, 2014).

Early studies of class I and class II methanol masers led to suggestions that they might be associated with different types of sources, however this turned out to be an observational bias in that some of the best-studied class I (e.g. Orion KL) and class II (e.g. W3(OH)) maser sources show no emission, or only very weak emission in the other class. Slysh et al. (1994) made a search for 44-GHz class I masers towards a sample of star formation regions known to host either $\mathrm{OH}$ maser and/or 6.7-GHz class II methanol masers. They detected many new class I methanol masers in this search and claimed an anticorrelation between the velocity ranges and the peak intensity of the class I and class II methanol masers in the same sources. Ellingsen (2005) searched for 95-GHz class I methanol masers towards a statistically complete sample of class II methanol masers, obtaining detections towards approximately 40 per cent of the sample, however Ellingsen found no evidence for the anticorrelations between the two classes claimed by Slysh et al.

The observations of Ellingsen were made with a single dish and so while they established the statistical relationship between the fraction of class II methanol maser sources which also host a class I methanol maser nearby, they did not show whether the two classes of methanol maser are driven by the same young high-mass star, nor the detailed spatial relationship between them. Here we report interferometric observations made with the Australia Telescope Compact Array (ATCA) of the 95-GHz class I methanol masers towards the sources detected by Ellingsen (2005). The locations of the class II methanol masers in all of these sources are known to sub-arcsecond precision from previous observations (Caswell et al. 2011; Green et al. 2012) and so we have the opportunity to make the first study of the relationship between class I and class II methanol masers at high resolution in a statistically complete sample.

\section{OBSERVATIONS}

The observations were undertaken with the ATCA between 2004 September 24 and 26 (project code C1273). The array was in the H75 configuration, with minimum and maximum baselines of 30.6 and $89.2 \mathrm{~m}$, respectively, and the synthesized beam width for the observations at $95-\mathrm{GHz}$ was approximately $6 \times 5$ arcsec. The correlator was configured with 256 spectral channels across a $32-\mathrm{MHz}$ bandwidth. At an observing frequency of $95-\mathrm{GHz}$, this corresponds to a total velocity range of $100 \mathrm{~km} \mathrm{~s}^{-1}$ with a velocity resolution of $0.47 \mathrm{~km} \mathrm{~s}^{-1}$ for uniform weighting of the lag function (a channel width of $0.4 \mathrm{~km} \mathrm{~s}^{-1}$ ).

The target sources were the detected $95-\mathrm{GHz}$ class I methanol masers from Ellingsen (2005). Ellingsen used the Mopra telescope to search for $95-\mathrm{GHz}$ class I methanol masers towards a statistically complete sample of $6.7-\mathrm{GHz}$ methanol masers in the Galactic longitude range $l=325^{\circ}-335^{\circ}, b= \pm 0.53^{\circ}$. We observed every source within the aforementioned Galactic longitude range that were observed to have 95-GHz emission in Ellingsen (2005). Not every source observed by Ellingsen was member of their statistically complete sample of $6.7-\mathrm{GHz}$ methanol masers. Therefore, 11 of the 32 sources reported here are not within the statistically complete sample due to them falling outside the coordinate/LSR velocity range or being excluded for reasons described in Ellingsen (2005). These 11 sources have been clearly noted in the observation tables, and are not included in analysis where a statistically complete sample is described.
The data were reduced with MIRIAD using the standard techniques for ATCA $3 \mathrm{~mm}$ spectral line observations. Amplitude calibration with respect to Uranus and PKS B1921-293 was observed as the bandpass calibrator. The data were corrected for atmospheric opacity and the absolute flux density calibration is estimated to be accurate to 20 per cent. This was estimated by comparing the measured flux density of the bandpass and phase calibrator sources over each of the $3 \mathrm{~d}$ of the observations. The observing strategy interleaved $5 \mathrm{~min}$ on-source for each of the maser targets with $2.5 \mathrm{~min}$ observations of a phase calibrator, alternating between two different phase calibrator sources 1600-44 and 1613-586. The data were self-calibrated (phase-only) using the emission from the brightest 95-GHz methanol maser in each pointing. After self-calibration we used continuum subtraction (modelled using the spectral channels without maser emission) to isolate the spectral line and continuum emission components.

The 95-GHz maser emission was then subsequently imaged with a velocity plane width of $0.25 \mathrm{~km} \mathrm{~s}^{-1}$. Imaging at higher than the spectral resolution of our observations does not affect our results due to the restrictions we place on determination of individual components. The pointing centre for each of the observations is listed in Table 1, along with information on the total time on-source for each source and the RMS noise for both the continuum data and a 0.25 $\mathrm{km} \mathrm{s}^{-1}$ spectral resolution image cube. We adopted a rest frequency of $95.169489-\mathrm{GHz}$ for the $8_{0}-7_{1} \mathrm{~A}^{+}$transition of methanol (Müller, Menten \& Mäder 2004).

\section{RESULTS}

We detected 95-GHz methanol maser emission towards all 32 of our target sources. A continuum source was detected in 14 out of 32 target pointings. The majority of continuum sources detected were point sources, however, more extended continuum emission was observed in four cases (G 326.641+0.61, G 331.132-0.24, G333.068-0.44, and G333.163-0.10). Table 2 contains details of the location and flux density of these continuum sources, along with information on whether the $6.7-\mathrm{GHz}$ pointing target or $95-\mathrm{GHz}$ class I masers were associated. An association was considered to exist between the $95-\mathrm{GHz}$ continuum emission and the two maser species, if the maser components were within $3 \mathrm{~s}$ of arc from the peak continuum emission.

In all observed sources the methanol maser emission was detected in many spectral channels. Emission in consecutive spectral channels is often at a similar location and is therefore considered a single component. In order to describe the maser emission as a series of 'spots, we have to define a set of criteria for when we consider emission adequately separated in space and velocity to be considered a unique maser component. Here we are considering emission regions to be independent if they are separated by at least 1.4 arcsec (upper $3 \sigma$ positional error across all sources) or separated by at least $1 \mathrm{~km} \mathrm{~s}^{-1}$ in LSR velocity. If the angular separation criteria were met, the component was considered unique; If only the velocity separation criterion was met, the spectrum of the component was closely considered in order to determine that it was unique, and not the result of broad emission from another component it shares a position with. The $95-\mathrm{GHz}$ components detected in the target sources are listed within Table 3. In total, 172 unique 95-GHz maser components were detected across our 32 class II targets. The absolute position of these components has been listed, along with their corresponding alphabetized location, as defined by Voronkov et al. (2014). 
Table 1. The coordinates of the pointing centre for each of the 6.7-GHz class II maser sites observed, along with on-source time and RMS noise for the observations. Distance estimates to the target sources along with their associated error (values in parenthesis are the negative error bars in cases where errors are asymmetrical) are also included and drawn from Green \& McClure-Griffiths (2011). Three sources (G 331.13-0.24, G 333.128-0.44, and G 333.466-0.16) did not have previously estimated linear distances, for these sources a near kinematic distance has been assumed using the same parameters and methodology presented in Green \& McClure-Griffiths (2011) and their distances denoted with an asterisk. The final column reports the linear offset between each class II pointing target and the closest detected 95-GHz maser component with velocity coincident to the velocity range of the class II emission (for the estimated distance). Velocity range values for the class II sources are drawn from Caswell et al. (2011) and Green et al. (2012).

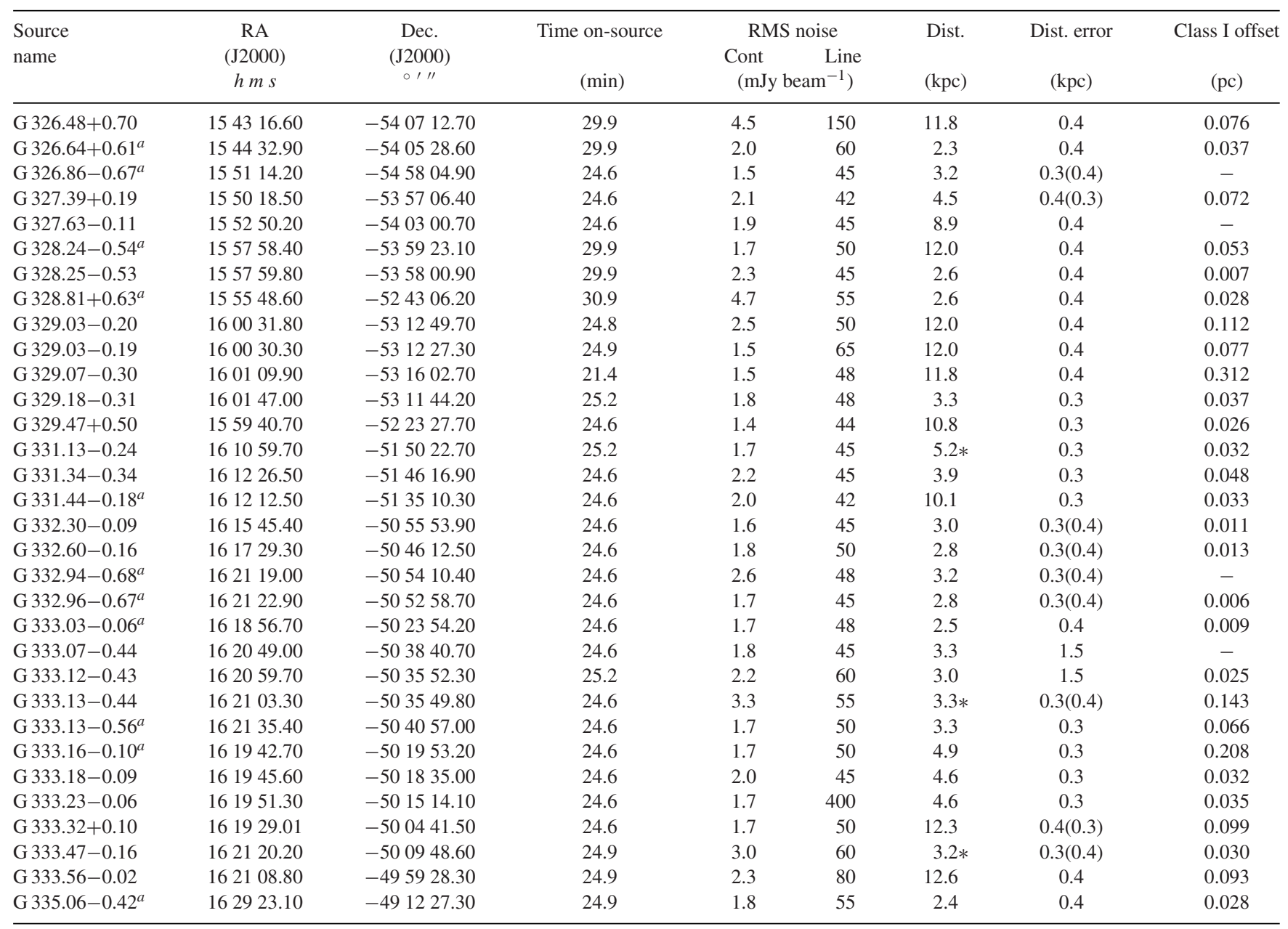

Note. ${ }^{a}$ Sources are not within the statistically complete sample of $6.7-\mathrm{GHz}$ methanol masers

We aim to compare our observations to those presented in Voronkov et al. (2014), who imaged 71 southern class I methanol maser sources at 36- and 44-GHz with the ATCA, with typical $1 \sigma$ RMS noise levels of $\sim 70$ and $\sim 120 \mathrm{mJy}$ at $36-$ and 44-GHz, respectively. Voronkov et al. identified class I components that were roughly spatially associated and assigned them a letter name for ease of reference. We have used these same locations for grouping and referring to the components presented here. The $95-\mathrm{GHz}$ components, along with the 36- and $44-\mathrm{GHz}$ components from Voronkov et al. (2014), have been plotted on a series of field images (see Fig. 1). There are 30 field images total, covering the 32 targets sources from our observations, as two fields have multiple pointings (G329.03-0.20 and G333.13-0.44). In addition to these field images, Fig. 2 shows scalar averaged spectra for all sources (except G328.25-0.53 where scalar averaging prevented emission from being clearly identifiable). These spectra all appear very similar to those presented in Ellingsen, Shabala \& Kurtz (2005) for the same sources, albeit with generally higher SNR.
In all but three instances, locations containing 95-GHz components also harbour 44-GHz emission (as specified by Voronkov et al. 2014). The exceptions occur in the sources G328.25-0.53 and $\mathrm{G} 328.81+0.63$, where the $95-\mathrm{GHz}$ masers are located within $\sim 4$ arcsec of a class II 6.7-GHz maser, but do not have an associated 44-GHz class I component.

A close association between $95-$ and $44-\mathrm{GHz}$ methanol masers is expected because the two transitions belong to the same transition series $\left(\mathrm{J}_{0}-(\mathrm{J}-1){ }_{1} A^{+}\right)$and there is a reported correlation between the observed flux density of the two transitions (Val'tts et al. 2000).

All, except one (G333.07-0.45), of our target sources had previously observed class I maser emission (from the $36-\mathrm{GHz}$ and 44-GHz transitions) reported by Voronkov et al. (2014). Due to the significantly smaller primary beam at $95-\mathrm{GHz}(\sim 30 \mathrm{arcsec})$, along with the expected flux density of the $95-\mathrm{GHz}$ masers being half of that observed in associated $44-\mathrm{GHz}$ masers, there are likely other 95-GHz components located outside the half-power range of our primary beam that we are unable to detect (specifically in regions with strong 44-GHz emission). 
Table 2. Details of the continuum sources detected in 14 of the pointing targets. Including location of the peak emission, peak flux density, integrated flux density and whether any association is observed with either class II 6.7-GHz masers or detected $95-\mathrm{GHz}$ masers.

\begin{tabular}{|c|c|c|c|c|c|c|c|}
\hline \multirow{2}{*}{$\begin{array}{l}\text { Source } \\
\text { name }\end{array}$} & \multirow{2}{*}{$\begin{array}{c}\text { RA } \\
(\mathrm{J} 2000) \\
h m s\end{array}$} & \multirow{2}{*}{$\begin{array}{c}\text { Dec. } \\
(\mathrm{J} 2000) \\
\circ, / 1\end{array}$} & \multirow{2}{*}{$\begin{array}{c}\text { Peak } \\
\text { flux density } \\
(\mathrm{mJy})\end{array}$} & \multirow{2}{*}{$\begin{array}{l}\text { RMS noise } \\
\text { (mJy) }\end{array}$} & \multirow{2}{*}{$\begin{array}{c}\text { Integrated } \\
\text { flux density } \\
(\mathrm{mJy})\end{array}$} & \multicolumn{2}{|c|}{ Associations } \\
\hline & & & & & & Class II & Class I \\
\hline G $326.48+0.70$ & $15: 43: 16.48$ & $-54: 07: 14.0$ & 94 & 4.3 & 105.2 & $\mathrm{y}$ & $\mathrm{y}$ \\
\hline $\mathrm{G} 326.64+0.61^{a}$ & $15: 44: 32.85$ & $-54: 05: 28.6$ & 23.2 & 2.1 & 41.9 & $\mathrm{y}$ & $\mathrm{y}$ \\
\hline G 326.86-0.67 & & & $<5$ & & & & \\
\hline G $327.39+0.19$ & & & $<7$ & & & & \\
\hline G 327.62-0.11 & & & $<6$ & & & & \\
\hline G $328.24-0.54^{a}$ & $15: 57: 58.37$ & $-53: 59: 21.9$ & 63.9 & 1.6 & 66.2 & $\mathrm{y}$ & $\mathrm{y}$ \\
\hline G $328.25-0.53$ & & & $<8$ & & & & \\
\hline $\mathrm{G} 328.81+0.63^{a}$ & $15: 55: 48.48$ & $-52: 43: 07.2$ & 973.3 & 4.8 & 1007 & $\mathrm{y}$ & $\mathrm{y}$ \\
\hline G $329.03-0.20$ & & & $<9$ & & & & \\
\hline G 329.03-0.19 & & & $<5$ & & & & \\
\hline G 329.07-0.30 & 16:01:07.86 & $-53: 16: 05.8$ & 9.6 & 1.6 & 9.7 & $\mathrm{n}$ & $\mathrm{n}$ \\
\hline & 16:01:09.99 & $-53: 16: 00.3$ & 7.4 & 1.6 & 7.2 & $\mathrm{y}$ & $\mathrm{n}$ \\
\hline G 329.18-0.31 & 16:01:47.99 & $-53: 11: 58.6$ & 9.4 & 1.9 & 11.6 & $\mathrm{n}$ & $\mathrm{n}$ \\
\hline & $16: 01: 47.22$ & $-53: 11: 41.3$ & 9.8 & 1.9 & 10.0 & $\mathrm{y}$ & $\mathrm{y}$ \\
\hline G $329.47+0.50$ & & & $<4$ & & & & \\
\hline G 331.13-0.24 & $16: 10: 59.67$ & $-51: 50: 23.8$ & 122.7 & 1.7 & 147.2 & $\mathrm{y}$ & $\mathrm{y}$ \\
\hline G 331.34-0.34 & & & $<7$ & & & & \\
\hline $\mathrm{G} 331.44-0.18^{a}$ & $16: 12: 12.75$ & $-51: 35: 10.4$ & 26.1 & 2.0 & 28.1 & $\mathrm{y}$ & $\mathrm{y}$ \\
\hline G 332.30-0.09 & $16: 15: 45.58$ & $-50: 55: 55.3$ & 17.9 & 1.6 & 27.4 & $\mathrm{y}$ & y \\
\hline G $332.60-0.16$ & & & $<7$ & & & & \\
\hline G 332.94-0.68 & & & $<9$ & & & & \\
\hline G $332.96-0.67^{a}$ & & & $<6$ & & & & \\
\hline G $333.03-0.06^{a}$ & & & $<6$ & & & & \\
\hline G 333.07-0.44 & $16: 20: 48.88$ & $-50: 38: 39.6$ & 11.6 & 1.8 & 30.6 & $\mathrm{y}$ & $\mathrm{y}$ \\
\hline G $333.12-0.43$ & $16: 20: 59.78$ & $-50: 35: 50.6$ & 14.0 & 2.4 & 14.0 & $\mathrm{y}$ & y \\
\hline G 333.13-0.44 & $16: 21: 02.46$ & $-50: 35: 57.1$ & 42.3 & 3.6 & 48.1 & $\mathrm{y}$ & $\mathrm{y}$ \\
\hline $\mathrm{G} 333.13-0.56^{a}$ & & & $<6$ & & & & \\
\hline G $333.16-0.10^{a}$ & $16: 19: 42.43$ & $-50: 19: 53.3$ & 16.0 & 1.8 & 30.7 & $\mathrm{y}$ & $\mathrm{y}$ \\
\hline G 333.18-0.09 & & & $<7$ & & & & \\
\hline G $333.23-0.06$ & 16:19:51.08 & $-50: 15: 13.9$ & 10.7 & 1.9 & 11.6 & $\mathrm{y}$ & $\mathrm{y}$ \\
\hline G $333.32+0.10$ & & & $<5$ & & & & \\
\hline G 333.47-0.16 & & & $<9$ & & & & \\
\hline G 333.56-0.02 & & & $<8$ & & & & \\
\hline $\mathrm{G} 335.06-0.42^{a}$ & & & $<6$ & & & & \\
\hline
\end{tabular}

NOte. ${ }^{a}$ Sources are not within the statistically complete sample of $6.7-\mathrm{GHz}$ methanol masers.

\subsection{Notes on individual sources}

With the exception of G333.07-0.44, all the sources reported here have previous high resolution observations of the 36- and 44-GHz transitions by Voronkov et al. (2014). In the comments below, we have not repeated the information given by Voronkov et al. relating to the general class I maser distribution, we comment only on the specifics of the new $95-\mathrm{GHz}$ methanol maser information.

The field images presented in this study are created in the same way as those previously presented by Voronkov et al. (2014) and therefore any prior comment on the Spitzer background images for individual sources will apply here.

\subsubsection{G326.48+0.70}

The majority of $95-\mathrm{GHz}$ methanol maser emission is associated with site A, close to the north-west class II 6.7-GHz maser. 95$\mathrm{GHz}$ components trace the same linear structure, beginning at $\mathrm{A}$ and extending south-west, observed in the 36- and 44-GHz transitions. Within the FWHM of the $95-\mathrm{GHz}$ primary beam, there are two EGOs, both considered likely outflow candidates (Cyganowski et al. 2008). There is an EGO located at A (obscured by maser symbols at this location in Fig. 1), along with a larger region several arcseconds to the east. Class I methanol maser emission is associated with both of these EGOs (only the western edge of the larger EGO), however, $95-\mathrm{GHz}$ methanol maser emission is only spatially associated with the EGO at A. In addition to these two EGOs, there is another infrared source with $4.5-\mu \mathrm{m}$ excess within the half-power range of our observations, located south-east of G. There is $3.5-\mathrm{mm}$ continuum source detected at $\mathrm{A}$, with peak intensity coincident with the densest cluster of class I methanol maser emission. 36- and 44-GHz emission has been detected in a second nearby $6.7-\mathrm{GHz}$ maser, located in the south-east of this field and associated with a strong infrared source. However, this region is far beyond the half-power point of our $95-\mathrm{GHz}$ pointing. A follow-up observation of this south-eastern class II source would likely provide additional detections of $95-\mathrm{GHz}$ class I methanol emission in this region.

\section{$3.1 .2 G 326.64+0.61$}

This 6.7-GHz target source is located on the edge of a large $8.0-\mu \mathrm{m}$ filament (seen in the south-east corner of the field). Class I emission in this field is widely spread, however, the half-power range of 
Table 3. Detailed list of the $95 \mathrm{GHz}$ components detected in each maser site, including their alphabetical location as defined by Voronkov et al. (2014), positions, and flux density. Source names have been converted from those of the individual pointings to those used by Voronkov et al. (2014); This results in two cases where two $95^{\circ} \mathrm{GHz}$ pointings have been combined into one source.

\begin{tabular}{|c|c|c|c|c|c|}
\hline $\begin{array}{l}\text { Source } \\
\text { name }\end{array}$ & Location & $\begin{array}{c}\text { LSR } \\
\text { velocity } \\
\left(\mathrm{km} \mathrm{s}^{-1}\right)\end{array}$ & $\begin{array}{c}\text { RA } \\
(\mathrm{J} 2000) \\
h m s\end{array}$ & $\begin{array}{l}\text { Dec. } \\
(\mathrm{J} 2000) \\
0,1 "\end{array}$ & $\begin{array}{c}\text { Flux } \\
\text { density } \\
\text { (Jy) }\end{array}$ \\
\hline \multirow[t]{9}{*}{ G $326.48+0.70$} & A & -47.0 & $15: 43: 16.67$ & $-54: 07: 16.9$ & 0.31 \\
\hline & A & -46.3 & $15: 43: 16.38$ & $-54: 07: 16.6$ & 0.33 \\
\hline & A & -45.2 & $15: 43: 16.49$ & $-54: 07: 14.5$ & 0.85 \\
\hline & A & -44.0 & $15: 43: 16.46$ & $-54: 07: 14.3$ & 6.04 \\
\hline & A & -41.7 & $15: 43: 16.37$ & $-54: 07: 15.1$ & 8.18 \\
\hline & A & -40.5 & $15: 43: 16.15$ & $-54: 07: 16.7$ & 14.62 \\
\hline & A & -37.7 & $15: 43: 16.22$ & $-54: 07: 16.1$ & 1.96 \\
\hline & $\mathrm{B}$ & -39.5 & $15: 43: 15.98$ & $-54: 07: 12.9$ & 3.56 \\
\hline & $\mathrm{B}$ & -39.3 & $15: 43: 15.84$ & $-54: 07: 11.3$ & 4.31 \\
\hline \multirow[t]{6}{*}{$\mathrm{G} 326.64+0.61^{a}$} & B & -43.0 & $15: 44: 33.60$ & $-54: 05: 20.9$ & 0.50 \\
\hline & B & -37.3 & $15: 44: 33.60$ & $-54: 05: 23.0$ & 1.57 \\
\hline & $\mathrm{D}$ & -41.0 & $15: 44: 32.96$ & $-54: 05: 31.0$ & 0.99 \\
\hline & $\mathrm{D}$ & -40.8 & $15: 44: 32.72$ & $-54: 05: 33.2$ & 1.27 \\
\hline & $\mathrm{D}$ & -40.0 & $15: 44: 32.95$ & $-54: 05: 30.8$ & 3.17 \\
\hline & $\mathrm{D}$ & -38.2 & $15: 44: 32.85$ & $-54: 05: 32.3$ & 6.45 \\
\hline \multirow[t]{6}{*}{ G 326.86-0.67 } & A & -67.3 & $15: 51: 13.84$ & $-54: 58: 04.7$ & 3.22 \\
\hline & A & -66.7 & $15: 51: 13.73$ & $-54: 58: 05.0$ & 8.31 \\
\hline & A & -66.0 & $15: 51: 13.76$ & $-54: 58: 05.1$ & 5.79 \\
\hline & A & -65.5 & $15: 51: 13.91$ & $-54: 58: 05.2$ & 1.44 \\
\hline & B & -68.8 & $15: 51: 14.11$ & $-54: 58: 03.8$ & 0.53 \\
\hline & $\mathrm{B}$ & -67.8 & $15: 51: 14.05$ & $-54: 58: 03.9$ & 2.04 \\
\hline \multirow[t]{3}{*}{ G $327.39+0.19$} & B & -88.8 & $15: 50: 18.53$ & $-53: 57: 06.3$ & 3.30 \\
\hline & B & -87.3 & $15: 50: 18.32$ & $-53: 57: 07.8$ & 1.96 \\
\hline & $\mathrm{B}$ & -86.5 & $15: 50: 18.13$ & $-53: 57: 07.4$ & 0.57 \\
\hline \multirow[t]{2}{*}{ G $327.62-0.11$} & A & -88.0 & $15: 52: 50.20$ & $-54: 03: 00.9$ & 6.78 \\
\hline & B & -86.8 & $15: 52: 50.31$ & $-54: 03: 05.5$ & 0.88 \\
\hline \multirow[t]{8}{*}{$\mathrm{G} 328.24-0.54^{a}$} & A & -42.3 & $15: 57: 58.38$ & $-53: 59: 22.9$ & 0.99 \\
\hline & A & -41.7 & $15: 57: 58.34$ & $-53: 59: 20.3$ & 1.12 \\
\hline & A & -40.8 & $15: 57: 58.27$ & $-53: 59: 17.7$ & 7.55 \\
\hline & A & -40.0 & $15: 57: 58.39$ & $-53: 59: 20.5$ & 0.42 \\
\hline & A & -39.3 & $15: 57: 58.55$ & $-53: 59: 19.5$ & 0.54 \\
\hline & A & -37.7 & $15: 57: 58.66$ & $-53: 59: 19.8$ & 0.42 \\
\hline & $\mathrm{B}$ & -44.0 & $15: 57: 58.46$ & $-53: 59: 31.3$ & 0.49 \\
\hline & $\mathrm{B}$ & -43.2 & $15: 57: 58.28$ & $-53: 59: 31.3$ & 1.86 \\
\hline \multirow[t]{3}{*}{ G $328.25-0.53$} & - & -44.0 & $15: 57: 59.93$ & $-53: 57: 57.4$ & 0.53 \\
\hline & - & -43.5 & $15: 57: 59.82$ & $-53: 58: 01.5$ & 0.47 \\
\hline & - & -41.7 & $15: 57: 59.81$ & $-53: 58: 00.5$ & 1.67 \\
\hline \multirow[t]{7}{*}{ G $328.81+0.63$} & A & -42.0 & $15: 55: 50.23$ & $-52: 43: 21.7$ & 4.72 \\
\hline & B & -42.5 & $15: 55: 49.15$ & $-52: 43: 23.6$ & 3.66 \\
\hline & $\mathrm{C}$ & -41.0 & $15: 55: 48.25$ & $-52: 43: 19.8$ & 2.46 \\
\hline & $\mathrm{C}$ & -40.3 & $15: 55: 48.49$ & $-52: 43: 19.5$ & 14.89 \\
\hline & $\mathrm{J}$ & -44.7 & $15: 55: 48.48$ & $-52: 43: 06.5$ & 1.22 \\
\hline & $\mathrm{J}$ & -43.5 & $15: 55: 48.53$ & $-52: 43: 05.8$ & 1.66 \\
\hline & $\mathrm{J}$ & -38.0 & $15: 55: 48.84$ & $-52: 43: 07.1$ & 0.50 \\
\hline \multirow[t]{8}{*}{$\mathrm{G} 329.03-0.20^{a b}$} & A & -46.5 & $16: 00: 30.33$ & $-53: 12: 28.7$ & 1.65 \\
\hline & $\mathrm{F}$ & -43.2 & $16: 00: 31.98$ & $-53: 12: 45.7$ & 18.87 \\
\hline & $\mathrm{F}$ & -42.0 & $16: 00: 31.84$ & $-53: 12: 47.2$ & 9.09 \\
\hline & G & -41.2 & $16: 00: 31.90$ & $-53: 12: 55.8$ & 3.67 \\
\hline & G & -40.5 & $16: 00: 31.76$ & $-53: 12: 51.5$ & 3.12 \\
\hline & G & -39.7 & $16: 00: 31.81$ & $-53: 12: 52.6$ & 3.13 \\
\hline & G & -38.2 & $16: 00: 32.05$ & $-53: 12: 53.7$ & 1.40 \\
\hline & G & -37.5 & $16: 00: 32.01$ & $-53: 12: 54.7$ & 4.15 \\
\hline \multirow[t]{4}{*}{ G $329.03-0.19^{b}$} & A & -46.5 & $16: 00: 30.29$ & $-53: 12: 28.6$ & 16.06 \\
\hline & A & -44.7 & $16: 00: 30.06$ & $-53: 12: 26.0$ & 1.09 \\
\hline & B & -45.5 & $16: 00: 30.45$ & $-53: 12: 13.5$ & 2.99 \\
\hline & $\mathrm{D}$ & -44.2 & $16: 00: 30.46$ & $-53: 12: 19.6$ & 0.67 \\
\hline \multirow[t]{3}{*}{ G 329.07-0.30 } & $\mathrm{D}$ & -41.7 & $16: 01: 08.95$ & $-53: 16: 07.8$ & 2.87 \\
\hline & $\mathrm{D}$ & -40.5 & 16:01:09.33 & $-53: 16: 03.5$ & 0.37 \\
\hline & $\mathrm{D}$ & -40.2 & $16: 01: 09.18$ & $-53: 16: 07.7$ & 0.37 \\
\hline
\end{tabular}


Table 3 - continued

\begin{tabular}{|c|c|c|c|c|c|}
\hline $\begin{array}{l}\text { Source } \\
\text { name }\end{array}$ & Location & $\begin{array}{c}\text { LSR } \\
\text { velocity } \\
\left(\mathrm{km} \mathrm{s}^{-1}\right)\end{array}$ & $\begin{array}{c}\text { RA } \\
(\mathrm{J} 2000) \\
h m s\end{array}$ & $\begin{array}{l}\text { Dec. } \\
(\mathrm{J} 2000) \\
0,11\end{array}$ & $\begin{array}{c}\text { Flux } \\
\text { density } \\
\text { (Jy) }\end{array}$ \\
\hline \multirow[t]{5}{*}{ G 329.18-0.31 } & $\mathrm{C}$ & -49.2 & $16: 01: 46.70$ & $-53: 11: 49.2$ & 0.56 \\
\hline & $\mathrm{E}$ & -50.2 & $16: 01: 47.26$ & $-53: 11: 43.8$ & 0.70 \\
\hline & $\mathrm{E}$ & -48.5 & 16:01:47.15 & $-53: 11: 42.1$ & 0.56 \\
\hline & $\mathrm{E}$ & -47.2 & $16: 01: 47.33$ & $-53: 11: 42.3$ & 2.54 \\
\hline & $\mathrm{E}$ & -46.2 & 16:01:47.17 & $-53: 11: 42.0$ & 0.39 \\
\hline \multirow[t]{5}{*}{ G $329.47+0.50$} & $\mathrm{~B}$ & -66.2 & $15: 59: 40.15$ & $-52: 23: 35.7$ & 2.64 \\
\hline & $\mathrm{E}$ & -69.0 & $15: 59: 40.66$ & $-52: 23: 27.5$ & 0.53 \\
\hline & $\mathrm{E}$ & -68.8 & $15: 59: 40.74$ & $-52: 23: 29.3$ & 0.53 \\
\hline & $\mathrm{E}$ & -67.3 & $15: 59: 40.69$ & $-52: 23: 28.4$ & 0.36 \\
\hline & $\mathrm{F}$ & -68.2 & 15:59:40.91 & $-52: 23: 30.4$ & 0.78 \\
\hline \multirow[t]{8}{*}{ G 331.13-0.24 } & A & -90.8 & $16: 10: 59.59$ & $-51: 50: 25.7$ & 35.43 \\
\hline & $\mathrm{A}$ & -86.8 & $16: 10: 59.74$ & $-51: 50: 24.5$ & 4.18 \\
\hline & $\mathrm{A}$ & -85.8 & $16: 10: 59.69$ & $-51: 50: 23.7$ & 4.35 \\
\hline & A & -84.8 & $16: 10: 59.73$ & $-51: 50: 24.3$ & 3.28 \\
\hline & A & -82.5 & $16: 10: 59.90$ & $-51: 50: 25.6$ & 2.67 \\
\hline & A & -80.3 & $16: 11: 00.00$ & $-51: 50: 26.7$ & 0.71 \\
\hline & $\mathrm{B}$ & -88.3 & $16: 11: 00.74$ & $-51: 50: 23.9$ & 10.42 \\
\hline & $\mathrm{C}$ & -84.0 & $16: 11: 00.25$ & $-51: 50: 25.9$ & 5.08 \\
\hline \multirow[t]{2}{*}{ G 331.34-0.34 } & A & -66.5 & $16: 12: 26.41$ & $-51: 46: 18.9$ & 0.60 \\
\hline & A & -65.2 & $16: 12: 26.50$ & $-51: 46: 20.6$ & 15.17 \\
\hline \multirow[t]{11}{*}{$\mathrm{G} 331.44-0.18^{a}$} & A & -91.3 & $16: 12: 12.28$ & $-51: 35: 13.3$ & 5.05 \\
\hline & A & -89.5 & $16: 12: 12.23$ & $-51: 35: 13.0$ & 1.02 \\
\hline & $\mathrm{C}$ & -87.3 & $16: 12: 11.41$ & $-51: 35: 18.3$ & 1.99 \\
\hline & $\mathrm{D}$ & -90.5 & $16: 12: 12.32$ & $-51: 35: 10.8$ & 0.28 \\
\hline & $\mathrm{D}$ & -90.3 & $16: 12: 12.35$ & $-51: 35: 12.3$ & 0.91 \\
\hline & $\mathrm{D}$ & -89.0 & $16: 12: 12.33$ & $-51: 35: 11.0$ & 0.75 \\
\hline & $\mathrm{D}$ & -88.5 & $16: 12: 12.44$ & $-51: 35: 09.6$ & 0.52 \\
\hline & $\mathrm{D}$ & -87.5 & $16: 12: 12.57$ & $-51: 35: 08.5$ & 3.21 \\
\hline & $\mathrm{D}$ & -86.5 & $16: 12: 12.55$ & $-51: 35: 08.6$ & 0.83 \\
\hline & $\mathrm{D}$ & -86.0 & $16: 12: 12.39$ & $-51: 35: 07.4$ & 0.44 \\
\hline & $\mathrm{D}$ & -85.5 & $16: 12: 12.51$ & $-51: 35: 09.2$ & 0.42 \\
\hline \multirow[t]{9}{*}{ G 332.30-0.09 } & A & -52.2 & $16: 15: 45.30$ & $-50: 55: 54.9$ & 0.31 \\
\hline & A & -51.7 & $16: 15: 45.72$ & $-50: 55: 55.0$ & 0.64 \\
\hline & A & -50.5 & $16: 15: 45.86$ & $-50: 55: 55.2$ & 5.71 \\
\hline & A & -49.2 & $16: 15: 45.78$ & $-50: 55: 55.3$ & 19.11 \\
\hline & $\mathrm{A}$ & -48.0 & $16: 15: 45.65$ & $-50: 55: 55.8$ & 7.21 \\
\hline & A & -47.2 & $16: 15: 45.45$ & $-50: 55: 55.7$ & 1.23 \\
\hline & A & -47.0 & $16: 15: 45.29$ & $-50: 55: 56.3$ & 1.16 \\
\hline & $\mathrm{A}$ & -46.5 & $16: 15: 45.45$ & $-50: 55: 54.3$ & 0.77 \\
\hline & $\mathrm{C}$ & -45.5 & $16: 15: 44.21$ & $-50: 55: 56.6$ & 1.50 \\
\hline \multirow[t]{2}{*}{ G 332.60-0.16 } & A & -46.2 & $16: 17: 29.27$ & $-50: 46: 14.6$ & 0.70 \\
\hline & $\mathrm{A}$ & -45.5 & $16: 17: 29.22$ & $-50: 46: 12.8$ & 8.52 \\
\hline \multirow[t]{3}{*}{ G 332.94-0.68 } & A & -50.0 & $16: 21: 19.32$ & $-50: 54: 13.7$ & 1.52 \\
\hline & A & -48.2 & $16: 21: 19.44$ & $-50: 54: 13.0$ & 4.34 \\
\hline & $\mathrm{A}$ & -46.7 & $16: 21: 19.32$ & $-50: 54: 13.8$ & 1.01 \\
\hline \multirow[t]{6}{*}{ G 332.96-0.67 } & A & -50.7 & $16: 21: 22.93$ & $-50: 52: 56.7$ & 0.42 \\
\hline & A & -50.2 & $16: 21: 22.71$ & $-50: 52: 55.7$ & 0.76 \\
\hline & A & -50.0 & $16: 21: 22.84$ & $-50: 52: 57.5$ & 0.49 \\
\hline & A & -48.7 & $16: 21: 22.88$ & $-50: 52: 58.7$ & 0.71 \\
\hline & A & -48.2 & $16: 21: 22.84$ & $-50: 52: 56.9$ & 2.06 \\
\hline & A & -46.7 & $16: 21: 22.76$ & $-50: 52: 56.7$ & 11.13 \\
\hline \multirow[t]{2}{*}{ G 333.03-0.06 } & $\mathrm{A}$ & -41.7 & $16: 18: 56.64$ & $-50: 23: 53.4$ & 1.17 \\
\hline & A & -40.3 & $16: 18: 56.77$ & $-50: 23: 54.9$ & 4.27 \\
\hline \multirow[t]{2}{*}{ G 333.07-0.44 } & - & -53.7 & $16: 20: 48.83$ & $-50: 38: 40.2$ & 0.47 \\
\hline & - & -51.2 & $16: 20: 48.64$ & $-50: 38: 39.1$ & 2.75 \\
\hline \multirow[t]{7}{*}{ G $333.13-0.56^{a}$} & A & -59.5 & $16: 21: 34.33$ & $-50: 41: 06.8$ & 1.13 \\
\hline & $\mathrm{B}$ & -57.2 & $16: 21: 35.91$ & $-50: 40: 49.1$ & 1.45 \\
\hline & $\mathrm{B}$ & -56.5 & $16: 21: 36.10$ & $-50: 40: 48.7$ & 1.74 \\
\hline & B & -56.3 & $16: 21: 36.33$ & $-50: 40: 48.3$ & 1.64 \\
\hline & $\mathrm{B}$ & -55.3 & $16: 21: 36.43$ & $-50: 40: 47.4$ & 4.65 \\
\hline & B & -54.0 & $16: 21: 36.08$ & $-50: 40: 48.1$ & 8.69 \\
\hline & $\mathrm{D}$ & -58.8 & $16: 21: 35.79$ & $-50: 40: 55.1$ & 7.51 \\
\hline
\end{tabular}


Table 3 - continued

\begin{tabular}{|c|c|c|c|c|c|}
\hline $\begin{array}{l}\text { Source } \\
\text { name }\end{array}$ & Location & $\begin{array}{c}\text { LSR } \\
\text { velocity } \\
\left(\mathrm{km} \mathrm{s}^{-1}\right)\end{array}$ & $\begin{array}{c}\text { RA } \\
(\mathrm{J} 2000) \\
h m s\end{array}$ & $\begin{array}{l}\text { Dec. } \\
(\mathrm{J} 2000) \\
0, " 1 "\end{array}$ & $\begin{array}{c}\text { Flux } \\
\text { density } \\
\text { (Jy) }\end{array}$ \\
\hline \multirow[t]{7}{*}{$\mathrm{G} 333.12-0.43^{c}$} & $\mathrm{~B}$ & -58.0 & $16: 20: 59.75$ & $-50: 35: 51.3$ & 0.32 \\
\hline & $\mathrm{B}$ & -56.3 & $16: 20: 59.71$ & $-50: 35: 51.7$ & 0.49 \\
\hline & B & -55.0 & $16: 20: 59.73$ & $-50: 35: 50.9$ & 1.43 \\
\hline & $\mathrm{B}$ & -52.3 & $16: 20: 59.49$ & $-50: 35: 50.5$ & 9.31 \\
\hline & B & -50.2 & $16: 20: 59.42$ & $-50: 35: 49.0$ & 8.10 \\
\hline & B & -49.3 & $16: 20: 59.63$ & $-50: 35: 50.2$ & 1.02 \\
\hline & B & -45.3 & $16: 20: 59.64$ & $-50: 35: 51.5$ & 1.74 \\
\hline \multirow{10}{*}{$\mathrm{G} 333.13-0.44^{c}$} & A & -48.7 & $16: 21: 02.58$ & $-50: 35: 54.5$ & 18.44 \\
\hline & A & -48.0 & $16: 21: 02.18$ & $-50: 35: 50.8$ & 18.85 \\
\hline & A & -47.7 & $16: 21: 02.41$ & $-50: 35: 53.0$ & 17.54 \\
\hline & A & -47.0 & $16: 21: 02.63$ & $-50: 35: 54.0$ & 19.87 \\
\hline & A & -46.2 & $16: 21: 02.35$ & $-50: 35: 53.1$ & 10.64 \\
\hline & A & -44.5 & 16:21:02.39 & $-50: 35: 52.9$ & 0.49 \\
\hline & $\mathrm{E}$ & -51.5 & $16: 21: 03.32$ & $-50: 35: 45.1$ & 0.41 \\
\hline & $\mathrm{E}$ & -50.7 & $16: 21: 03.16$ & $-50: 35: 45.9$ & 1.91 \\
\hline & $\mathrm{DF}$ & -49.7 & $16: 21: 03.73$ & $-50: 35: 38.8$ & 12.21 \\
\hline & $\mathrm{F}$ & -51.2 & $16: 21: 03.18$ & $-50: 35: 38.4$ & 0.90 \\
\hline \multirow[t]{2}{*}{ G 333.16-0.10 } & A & -92.0 & $16: 19: 42.12$ & $-50: 20: 00.2$ & 0.93 \\
\hline & B & -91.0 & $16: 19: 42.71$ & $-50: 19: 55.3$ & 4.74 \\
\hline \multirow[t]{3}{*}{ G 333.18-0.09 } & A & -86.8 & $16: 19: 45.54$ & $-50: 18: 32.4$ & 0.80 \\
\hline & A & -86.3 & $16: 19: 45.65$ & $-50: 18: 33.6$ & 3.25 \\
\hline & A & -85.3 & $16: 19: 45.96$ & $-50: 18: 31.9$ & 0.68 \\
\hline \multirow[t]{4}{*}{ G 333.23-0.06 } & A & -89.5 & $16: 19: 49.52$ & $-50: 15: 14.1$ & 0.89 \\
\hline & A & -86.8 & $16: 19: 49.49$ & $-50: 15: 14.3$ & 35.57 \\
\hline & $\mathrm{C}$ & -85.0 & $16: 19: 51.41$ & $-50: 15: 14.1$ & 4.90 \\
\hline & $\mathrm{C}$ & -83.8 & $16: 19: 51.28$ & $-50: 15: 12.5$ & 0.40 \\
\hline \multirow[t]{8}{*}{ G $333.32+0.10$} & A & -49.2 & $16: 19: 28.74$ & $-50: 04: 38.7$ & 1.11 \\
\hline & A & -45.5 & $16: 19: 29.26$ & $-50: 04: 43.6$ & 0.38 \\
\hline & A & -44.0 & $16: 19: 28.85$ & $-50: 04: 40.7$ & 3.78 \\
\hline & $\mathrm{B}$ & -48.0 & $16: 19: 28.14$ & $-50: 04: 38.7$ & 0.86 \\
\hline & B & -47.2 & $16: 19: 28.34$ & $-50: 04: 39.1$ & 0.56 \\
\hline & $\mathrm{B}$ & -46.7 & $16: 19: 27.98$ & $-50: 04: 42.8$ & 0.56 \\
\hline & B & -46.0 & 16:19:28.09 & $-50: 04: 37.4$ & 0.47 \\
\hline & $\mathrm{D}$ & -44.7 & $16: 19: 28.62$ & $-50: 04: 53.5$ & 0.73 \\
\hline \multirow{6}{*}{ G $333.47-0.16$} & A & -45.0 & $16: 21: 19.94$ & $-50: 09: 42.0$ & 3.19 \\
\hline & $\mathrm{B}$ & -41.0 & $16: 21: 20.10$ & $-50: 09: 38.1$ & 2.13 \\
\hline & $\mathrm{C}$ & -42.3 & $16: 21: 20.55$ & $-50: 09: 55.5$ & 1.80 \\
\hline & $\mathrm{D}$ & -43.0 & $16: 21: 20.15$ & $-50: 09: 46.7$ & 5.38 \\
\hline & $\mathrm{D}$ & -40.5 & $16: 21: 20.42$ & $-50: 09: 48.8$ & 0.43 \\
\hline & $\mathrm{E}$ & -42.5 & $16: 21: 19.69$ & $-50: 09: 33.7$ & 1.42 \\
\hline \multirow[t]{3}{*}{ G 333.56-0.02 } & A & -40.8 & $16: 21: 08.77$ & $-49: 59: 49.5$ & 0.81 \\
\hline & A & -39.5 & $16: 21: 08.74$ & $-49: 59: 49.5$ & 9.35 \\
\hline & A & -38.2 & $16: 21: 08.59$ & $-49: 59: 47.8$ & 0.73 \\
\hline \multirow[t]{8}{*}{ G $335.06-0.42^{a}$} & A & -41.7 & $16: 29: 23.50$ & $-49: 12: 23.2$ & 1.13 \\
\hline & A & -41.2 & $16: 29: 23.46$ & $-49: 12: 22.5$ & 0.57 \\
\hline & A & -40.5 & $16: 29: 23.42$ & $-49: 12: 21.0$ & 3.70 \\
\hline & B & -39.7 & $16: 29: 22.98$ & $-49: 12: 32.8$ & 0.31 \\
\hline & B & -38.8 & $16: 29: 22.80$ & $-49: 12: 32.4$ & 4.83 \\
\hline & B & -37.7 & $16: 29: 23.04$ & $-49: 12: 32.1$ & 3.25 \\
\hline & B & -37.3 & $16: 29: 23.07$ & $-49: 12: 30.5$ & 0.53 \\
\hline & B & -37.0 & $16: 29: 23.19$ & $-49: 12: 29.4$ & 0.47 \\
\hline
\end{tabular}

Note. ${ }^{a}$ Sources are not within the statistically complete sample of 6.7-GHz methanol masers.

${ }^{b}$ Components from this target are included in the multipointing field image G 329.03-0.20.

${ }^{c}$ Components from this target are included in the multipointing field image G 333.13-0.44.

our observations contains the majority of locations of previously detected methanol maser emission (A, B, D, E, G, and J). 95-GHz maser emission is observed in two locations (B and D). A 3.5-mm continuum source is detected near $\mathrm{D}$ and $\mathrm{E}(\sim 3 \operatorname{arcsec}$ north-west of the 6.7-GHz maser), with the majority of class I maser emission at $\mathrm{D}$ associated with the southern edge. Additionally the $95-\mathrm{GHz}$ masers at this location are arranged in a line extending from the edge of this continuum emission towards the south-west. 


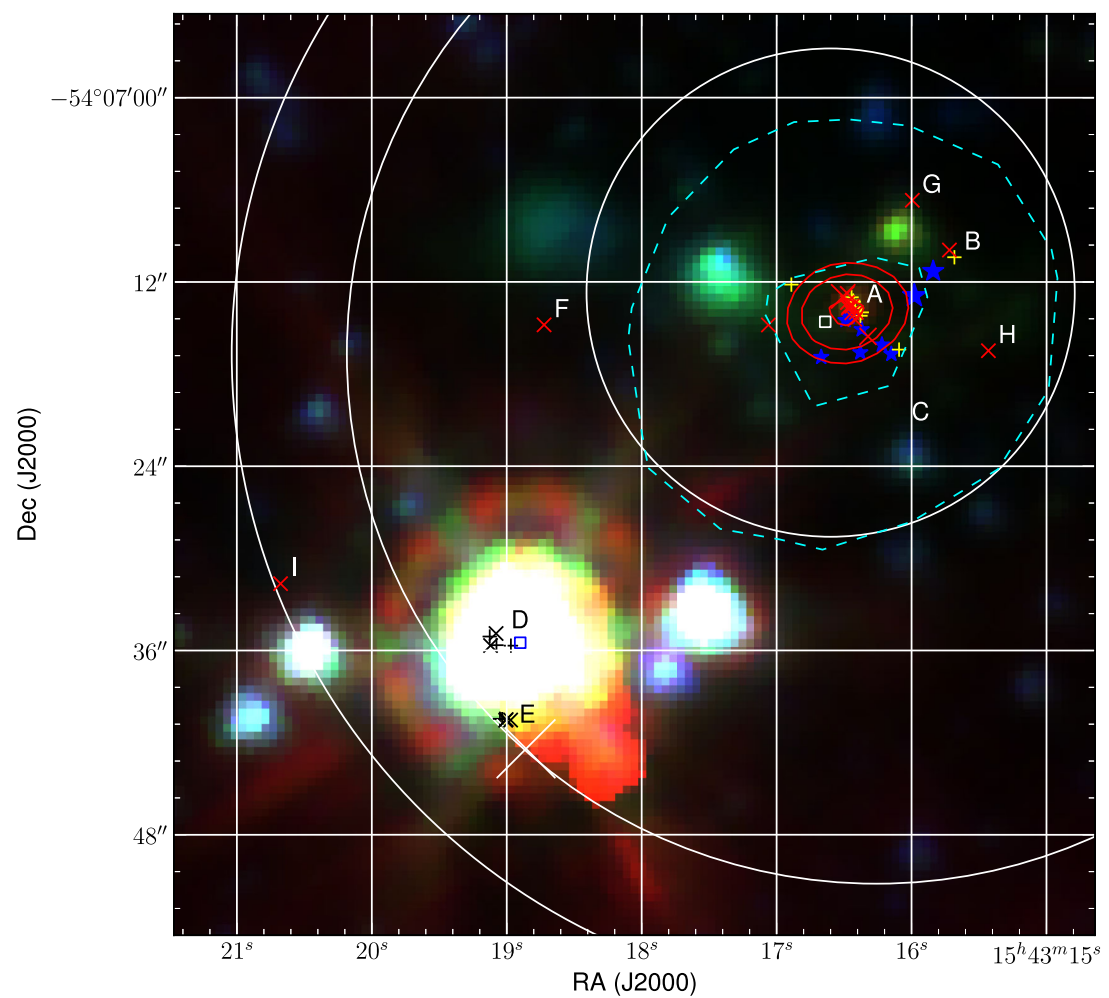

Figure 1. Field image for G $326.48+0.70$ and field images for the remaining 29 class I sources are available online. Four different symbols are projected on the Spitzer background images, a square symbol represents a 6.7-GHz class II methanol maser, and the cross, plus, and star symbols represent 36-, 44-, and 95-GHz class I maser emission, respectively. Solid contours on the images represent 95-GHz continuum emission where applicable. Dashed contours represent the 50 per cent and 90 per cent levels of the ATLASGAL emission. The white circles, in order of increasing size, represent the full width at half-maximum (FWHM) of the primary beam and are centred on the pointing direction for the 95-, 44-, and 36-GHz observations.

\section{$3.1 .36326 .86-0.68$}

Class I emission is relatively tightly clustered in this source, with locations $\mathrm{B}$ and A containing all the observed $95-\mathrm{GHz}$ maser components. The class I emission at $\mathrm{B}$ is associated with a $6.7-\mathrm{GHz}$ class II maser. East of the class II maser is a dark cloud, with class I emission at $\mathrm{B}$ and $\mathrm{C}$ located at the interface of this region (Peretto \& Fuller 2009). The 95-GHz masers in this source are located at the south-east edge of an EGO (likely outflow candidate according to Cyganowski et al. 2008).

\section{1 .4 G327.39+0.20}

The methanol maser emission is separated into two separate locations (A and B), both with 6.7-GHz masers and associated with strong infrared sources. The $95-\mathrm{GHz}$ pointing was centred on the westernmost 6.7-GHz maser (327.392+0.199) within this field. Although both locations are within the FWHM of the $95-\mathrm{GHz}$ primary beam, $95-\mathrm{GHz}$ maser emission is only observed at $\mathrm{B}$. The infrared source associated with B also contains a $4.5-\mu \mathrm{m}$ halo that has been classified as an EGO and considered a possible outflow candidate in Cyganowski et al. (2008).

\subsubsection{G327.62-0.11}

The centre of this field contains a strong infrared source where the $6.7-\mathrm{GHz}$ maser target and class I maser location A is situated. Only two $95-\mathrm{GHz}$ components were observed in this source, one component tightly co-located with the $6.7-\mathrm{GHz}$ maser, and one component at B on the southern edge of the infrared source. Similar to what is observed in the other class I transitions, the 95-GHz maser emission at location $\mathrm{A}$ is considerably stronger than that observed in B (Voronkov et al. 2014).

\section{1 .6 G328.24-0.55}

The 6.7-GHz pointing target is in the south-western corner of the field, with the majority of class I maser emission located nearby in $\mathrm{A}$ and $\mathrm{B}$. The $95-\mathrm{GHz}$ masers at A are loosely clustered, with the majority of these components being associated with other class I maser emission. The 6.7-GHz maser is a few arcseconds south of A and associated with a 95-GHz component. A 3.5-mm continuum source is observed, co-spatially associated with the class II $6.7-\mathrm{GHz}$ maser. The $95-\mathrm{GHz}$ masers at A appear to follow some sort of arc that may be related to the $8.0-\mu \mathrm{m}$ PDR situated north-east of these masers.

\section{1 .7 G328.25-0.53}

The class II 6.7-GHz maser in this source is associated with a strong infrared source in the south-west of the field. The 36- and 44-GHz emission in this source is spread between six locations spanning over 1 arcmin squared, only one of which (A) is within the FWHM of the $95-\mathrm{GHz}$ primary beam. The $95-\mathrm{GHz}$ masers in this source are not associated with any of the locations where 36- and 44$\mathrm{GHz}$ masers are detected, and instead they are associated with the 6.7-GHz maser target. This is one of only two cases across all of our sources where $95-\mathrm{GHz}$ masers are observed offset from the other 


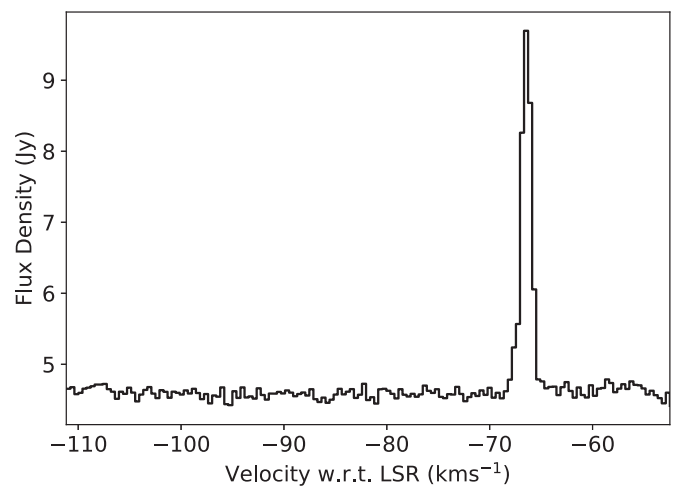

(a) G326.859-0.67

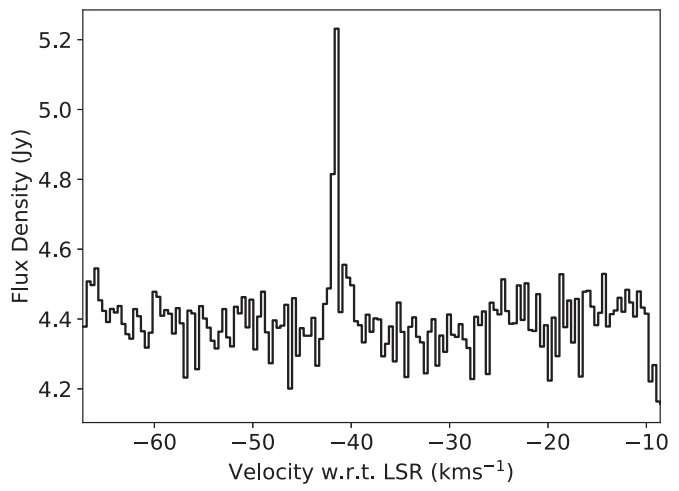

(c) G329.066-0.30

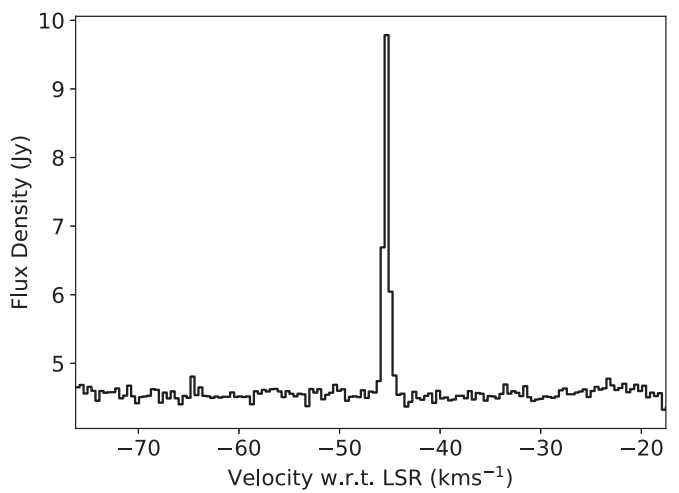

(e) G332.604-0.16

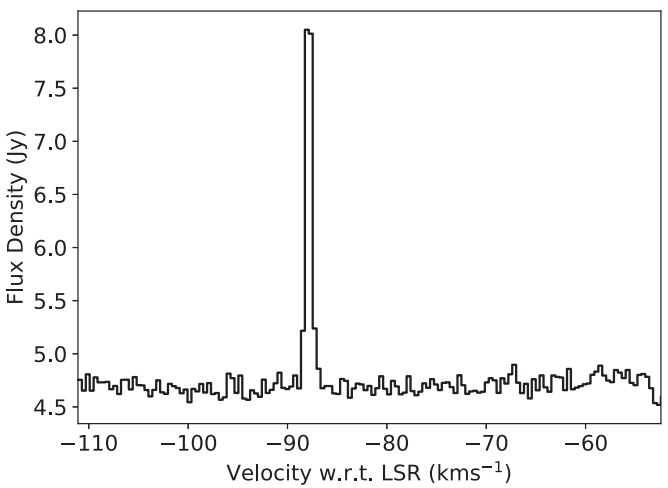

(b) G327.618-0.11

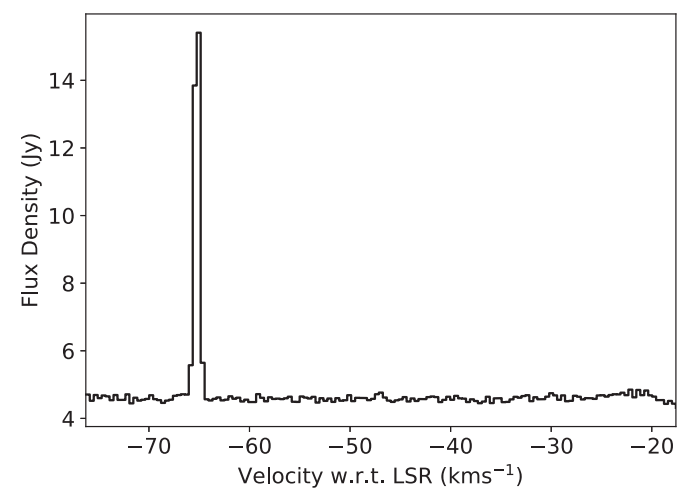

(d) G331.342-0.34

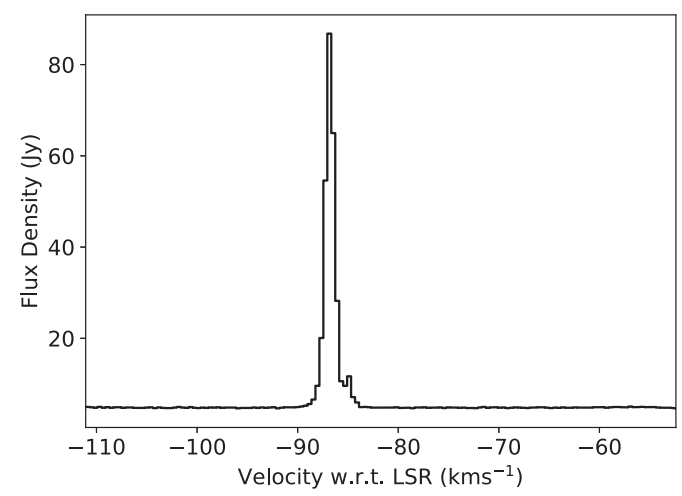

(f) G333.234-0.06

Figure 2. Representative spectra of $95-\mathrm{GHz}$ class I methanol masers from the current observations. Spectra for the remaining sources in the sample are available online. The spectra were extracted from scalar averaging of the self-calibrated ultraviolet (UV)-data used to create spectral line image cubes. Scalar averaged spectra allow us to see maser emission from all class I locations in the primary beam, but have a positive offset baseline because of contributions from the system temperature of the receivers and any continuum emission in the primary beam.

transitions of class I emission. An EGO is also nearby to this class II source (likely outflow candidate according to Cyganowski et al. 2008), with one 95-GHz component located at the interface of the EGO and the infrared source.

\subsubsection{G328.81+0.63}

Two strong class II 6.7-GHz masers, separated by 2.'5, are projected on to a $\mathrm{H}_{\text {II }}$ region in the centre of this field (Walsh et al. 1998;
Green et al. 2012). The 95-GHz maser emission is spatially associated with the two 6.7-GHz masers at J. A 3.5-mm continuum source is detected at $\mathrm{J}$, with peak emission at the location of the western 6.7$\mathrm{GHz}$ maser. Interestingly there is no $44-\mathrm{GHz}$ maser emission associated with the $95-\mathrm{GHz}$ masers in this location, however, indicating the continuum source may be seeding the emission of these masers. This may occur due to the 95-GHz masers amplifying the underlying continuum emission, which may differ significantly between 7- and 3.5-mm, which boosts the expected ratio of $95-44-\mathrm{GHz}$ 
flux densities at these projected locations. $95-\mathrm{GHz}$ masers at $\mathrm{B}$ and $\mathrm{C}$ trace the southern edge of the diffuse $8.0-\mu \mathrm{m}$ excess south of the $\mathrm{H}$ II region.

\section{1 .9 G329.03-0.20}

This field contains two separate $95-\mathrm{GHz}$ pointings, one centred on the north-western 6.7-GHz maser 329.031-0.198 and the other on the south-eastern 6.7-GHz maser 329.029-0.205. Class I maser emission in the south-east pointing is organized in a linear structure (from $\mathrm{F}$ to $\mathrm{H}$ ), extending north and south from the class II pointing target. The majority of the $95-\mathrm{GHz}$ emission in the north-west pointing is clustered about the $6.7-\mathrm{GHz}$ maser (location A). An EGO is present in this field (classified as likely outflow candidate by Cyganowski et al. 2008), several arcseconds south of A, with a single $36-\mathrm{GHz}$ component located on its western edge.

\subsubsection{G329.07-0.31}

The class II 6.7-GHz maser target is located in the centre of a strong infrared source. An additional strong infrared source is on the eastern edge of the FWHM of the $95-\mathrm{GHz}$ primary beam. Extended $4.5-\mu \mathrm{m}$ emission is present near both of the infrared sources, with both being classified as possible outflow candidates by Cyganowski et al. (2008). All detected $95-\mathrm{GHz}$ maser components are located south-west of the 6.7-GHz maser, at the interface of the EGO between the western infrared source and location D. Interestingly we see the second case of a $95-\mathrm{GHz}$ maser being offset from any previously observed class I maser emission, with the maser spot associated with the western edge of the infrared source. There are two weak continuum sources detected in this field, the weaker continuum source is spatially associated with the $6.7-\mathrm{GHz}$ maser, and the stronger (40 per cent brighter) continuum source is located north-west of $\mathrm{D}$, with no associated methanol emission or infrared source.

\subsubsection{G329.18-0.31}

The 6.7-GHz maser target's location is projected on to a prominent EGO (likely outflow candidate according to Cyganowski et al. 2008). The $95-\mathrm{GHz}$ masers at $\mathrm{E}$ appear to trace the eastern boundary of the YSO and the EGO. The 95-GHz component at $\mathrm{C}$ is also located on the south-western edge of the EGO. Two weak continuum sources are detected in this region. The northern-most is positionally associated with the class I emission and 6.7-GHz maser at E. The southern-most continuum source is outside the FWHM of the 95-GHz primary beam with no visible associations. Both locations $\mathrm{D}$ and $\mathrm{F}$ contain comparatively bright $44-\mathrm{GHz}$ class I methanol masers and are within the half-power range of the $95-\mathrm{GHz}$ primary beam, however, no $95-\mathrm{GHz}$ emission was detected in either of these regions.

\section{$3.1 .12 G 329.47+0.50$}

The majority of class I maser emission is clustered around the 6.7-GHz maser in the centre of the field. This class II source is on the eastern edge of an EGO (classified as a likely outflow candidate by Cyganowski et al. 2008). The majority of 95-GHz maser emission in this source is arranged in a line extending from the position of the $6.7-\mathrm{GHz}$ maser towards the south-east.

\section{$3.1 .13 G 331.13-0.24$}

The 6.7-GHz source is located on the north-eastern edge of one of the northern-most $\mathrm{H}$ II regions in the field. This class II source is positioned at the interface between an EGO (likely outflow candidate according to Cyganowski et al. 2008) and this $\mathrm{H}$ II region. 95-GHz masers are located at the three locations nearby to this $\mathrm{H}$ II region. The 95-GHz maser components are organized into a curve starting at the position of the 6.7-GHz maser, going through $\mathrm{C}$ and ending to the east at location B. A $3.5-\mathrm{mm}$ continuum source is detected, encompassing the entire northern $\mathrm{H}$ II region, including the majority of $95-\mathrm{GHz}$ emission.

\subsubsection{G331.34-0.35}

A strong infrared source dominates the centre of this compact class I maser. The 6.7-GHz maser is situated centrally to this infrared source. A class I masing component with emission from 36-, 44-, and $95-\mathrm{GHz}$ is situated at the interface between the strong infrared source and an EGO (classified as a possible outflow candidate by Cyganowski et al. 2008). We observe another component at $95 \mathrm{GHz}$ to the north-west, closer to the location of the $6.7-\mathrm{GHz}$ maser.

\section{1 .15 G331.44-0.19}

The 6.7-GHz class II target source is projected on to the edge of a compact $\mathrm{H}$ II region. 95-GHz masers are detected at all three locations (A, C, and D) that fall within the half power point of the $95-\mathrm{GHz}$ primary beam. A second $6.7-\mathrm{GHz}$ maser is also located within the FWHM of the $95-\mathrm{GHz}$ primary beam at C. $95-\mathrm{GHz}$ emission in $\mathrm{A}$ and $\mathrm{D}$ trace a line across the $\mathrm{H}$ II region, connecting the two locations. The majority of 36- and 44-GHz components reported by Voronkov et al. (2014) form a line extending to the south-west from A through to E. Due to the line of $95-\mathrm{GHz}$ components connecting $\mathrm{D}$ and $\mathrm{A}$, class I maser emission from locations A through E may be all part of the same linear structure. A 3.5-mm continuum source is detected near D, with the emission between D and A tracing the outer edge of the emission.

\subsubsection{G332.30-0.09}

Two closely separated $6.7-\mathrm{GHz}$ masers are within this field, the western 6.7-GHz maser (332.2950.094, the pointing target for this source), is located at the southern edge of an EGO (possible outflow candidate according to Cyganowski et al. 2008), and the second maser (332.296-0.094) is approximately 3 arcsec to the east. All but one of the $95-\mathrm{GHz}$ maser components in this source are clustered between the northern edge of a $\mathrm{H}$ II region and the EGO to the north-west, with the other component situated $\sim 15$ arcsec west. The 95-GHz components close to the eastern 6.7-GHz maser appear to be systematically offset by $\sim 1$ arcsec south of the nearby $44-\mathrm{GHz}$ maser components. An elongated 3.5-mm continuum source is detected at the location of the two 6.7-GHz masers, and encompasses all the $95-\mathrm{GHz}$ maser emission at $\mathrm{A}$.

\section{$3.1 .17 G 332.60-0.17$}

There are two sites of class I methanol emission within this source. The 6.7-GHz maser is associated with the southern class I emission and a faint EGO (classified as likely outflow by Cyganowski et al. 2008). Both 95-GHz maser components are situated on the western edge of this EGO, nearby (within 2 arcsec) to the class II maser. 


\subsubsection{G332.94-0.69}

The class II 6.7-GHz maser is located on the eastern edge of an infrared source, surrounded by an EGO (classified as likely outflow candidate by Cyganowski et al. 2008) extending towards the southeast. All 95-GHz masers are situated at location A within the southeastern region of the EGO. No $95-\mathrm{GHz}$ maser emission is detected in location $\mathrm{B}$, despite the relative brightness of the detected $44-\mathrm{GHz}$ components.

\subsubsection{G332.96-0.68}

Location A, at the northern edge of a infrared point source with associated EGO (likely outflow candidate according to Cyganowski et al. 2008), contains the majority of class I emission in this source. The EGO extends to the south-east, ending near B. The $6.7-\mathrm{GHz}$ target maser is projected on this infrared point source, with the bulk of the observed 95-GHz emission clustered closely associated.

\subsubsection{G333.03-0.06}

The 6.7-GHz maser along with class I emission in this source is tightly clustered between a strong infrared source and a region of 4.5- $\mu \mathrm{m}$ excess (not classified as EGO by Cyganowski et al. 2008). The 95-GHz masers here have a close association with the $44-\mathrm{GHz}$ maser components.

\subsubsection{G333.07-0.45}

This is the source with no previous observation made by Voronkov et al. (2014). The 6.7-GHz maser pointing target is projected on to the centre of a bright infrared region, surrounded by a region of 4.5$\mu \mathrm{m}$ excess. There are two detected $95-\mathrm{GHz}$ masers in this source, both offset to the north-west of the class II maser. An elongated $3.5-\mathrm{mm}$ continuum source is detected, with peak emission colocated with the $6.7-\mathrm{GHz}$ masers and an extension towards the south-east.

\subsubsection{G333.13-0.44}

Four $6.7-\mathrm{GHz}$ class II masers are present in this field, with two separate $95-\mathrm{GHz}$ pointings covering the densest regions of class I emission (targets 333.128-0.440 in the east and 333.121-0.434 in the west). The half-power range of the eastern pointing contains four distinct regions of class I emission (A, D, E, and F), with a secondary $6.7-\mathrm{GHz}$ maser associated with a strong infrared source at A. All $95-\mathrm{GHz}$ masers from the eastern pointing form a line, starting at the position of the $6.7-\mathrm{GHz}$ maser at $\mathrm{A}$ and extending towards the north-west. A 3.5-mm continuum source is also detected a few arcseconds south-west of this secondary 6.7-GHz maser.

The western pointing contains a single compact region of class I emission, clustered about the 6.7-GHz maser target (B), and located near the edge of a dark cloud. A 3.5-mm continuum source is also detected at this location, encompassing the 6.7-GHz maser and all but one of the $95-\mathrm{GHz}$ maser components at $\mathrm{B}$.

\section{$3.1 .23 G 333.13-0.56$}

The pointing target for this class I source was the southern-most of the two closely spaced 6.7-GHz masers, $333.128-0.560 .95-\mathrm{GHz}$ masers are seen at three separate locations within this source, with the majority associated with an EGO (classified as possible outflow candidate by Cyganowski et al. 2008) north-east of the northern 6.7-GHz maser (B). These 95-GHz components appear to be distributed in a line across the EGO. Bright $95-\mathrm{GHz}$ emission is also observed at D, equidistant from both class II masers. Emission from all three different class I transitions is projected at this location (D), with the masers potentially being associated with a slight $4.5-\mu \mathrm{m}$ excess. Voronkov et al. (2014) suggested that B and D might be connected with a curved $4.5-\mu \mathrm{m}$ structure going through the nearby 6.7-GHz maser.

\section{1 .24 G333.16-0.10}

6.7-GHz maser is associated with the eastern side of a strong infrared source, and $\mathrm{H}_{\text {II }}$ region in the centre of the field. Class I maser emission is observed in two compact locations (A and B) in this source. The masers at $\mathrm{B}$ are close to the $6.7-\mathrm{GHz}$ maser ( $\sim 2 \operatorname{arcsec}$ ), whereas class I emission at A is offset to the south of the infrared source. An elongated 3.5-mm continuum source is detected, encompassing the infrared source and class I and class II emission at B.

\subsubsection{G333.18-0.09}

The 6.7-GHz maser target is associated with an infrared point source surround by a prominent EGO (classified as likely outflow candidate by Cyganowski et al. 2008) extending to the south-east. The 95-GHz maser components are spatially associated with the location of previously reported $36-$ and $44-\mathrm{GHz}$ components at A along the northern edge of the EGO.

\subsubsection{G333.23-0.06}

The south-eastern 6.7-GHz maser (333.234-0.062) is the pointing target of the $95-\mathrm{GHz}$ observations in this source. A secondary 6.7-GHz maser (333.234-0.060) is located a few arcseconds northwest, and is associated with a bright infrared source. 95-GHz emission is distributed between two locations, A and C. The former is outside the FWHM of the $95-\mathrm{GHz}$ primary beam, however, it contains a very bright $95-\mathrm{GHz}$ component $(35.57 \mathrm{Jy}$, strong relative to the typical $95-\mathrm{GHz}$ flux density values we observe). The 95-GHz masers at region $\mathrm{C}$ have a close spatial association with the position of the south-eastern $6.7-\mathrm{GHz}$ maser. $3.5-\mathrm{mm}$ continuum emission is detected, situated between the two $6.7-\mathrm{GHz}$ masers on the south-eastern edge of the infrared source.

\section{$3.1 .276333 .32+0.11$}

The $6.7-\mathrm{GHz}$ maser in this field is projected on to a strong infrared source, with an EGO (classified as likely outflow candidate by Cyganowski et al. 2008) at the north-western edge. $95-\mathrm{GHz}$ maser components at location A are projected across the infrared source, with one component of $95-\mathrm{GHz}$ emission located significantly south-east of any previously reported class I emission. Interestingly in this source there does not appear to be a close spatial association between the 95-GHz components and the 36- and 44-GHz components. This may be due to evolution in the source, over the 2.5 yr between observations. 


\section{1 .28 G333.47-0.16}

The 6.7-GHz maser pointing target in this source is located within the southern half of an EGO (classified likely outflow candidate by Cyganowski et al. 2008). Voronkov et al. (2014) note a curved distribution of class I emission at locations A, B, D, and F tracing a bow shock with the $6.7-\mathrm{GHz}$ maser situated at the apex. The 95-GHz masers are consistent with the positions of the previously observed class I masers at A and B. However, at D one $95-\mathrm{GHz}$ maser is located with the previously observed emission, and another is observed east of the $6.7-\mathrm{GHz}$ maser at the edge of the EGO. No 95-GHz masers are observed at $\mathrm{F}$, possibly due to the $44-\mathrm{GHz}$ maser here being relatively weak compared to other locations in this source. 95-GHz masers are located at $\mathrm{E}$ and $\mathrm{C}$ also, making it appear that these masers trace the entire eastern edge of the EGO.

\subsubsection{G333.56-0.02}

A mistake in the observations of this source caused the pointing to be $\sim 18$ arcsec north of the intended 6.7-GHz maser target. Therefore, all the class I emission is outside the FWHM of the 95-GHz primary beam to the south. All class I maser emission in this source is compactly clustered about this $6.7-\mathrm{GHz}$ class II maser. One of the 95-GHz masers is offset to the north-east from the other class I emission.

\subsubsection{G335.06-0.43}

The class II maser is within the central region of an EGO (classified as a likely outflow candidate by Cyganowski et al. 2008). The 95-GHz masers at B and D closely trace the southern edge of the EGO. Voronkov et al. note that the class I emission at A appears to trace the northern edge of the EGO and the $95-\mathrm{GHz}$ components at this location are consistent with this observation.

\section{DISCUSSION}

Throughout our discussion we will directly compare the 36- and 44-GHz methanol maser properties presented by Voronkov et al. (2014) to the current $95-\mathrm{GHz}$ data. However, before doing that, it is important to highlight the differences in sample selection between the Voronkov et al. study and our investigation. Voronkov et al. selected almost all southern sources with previously detected class I maser emission from the single-dish searches of Slysh et al. (1994); Val'tts et al. (2000); Ellingsen (2005). The target sources for these original single-dish observations were highly varied, with a mix of known 6.7-GHz class II masers and $\mathrm{H}_{\text {II }}$ regions. Comparatively, the targets for our investigation are based on the Ellingsen (2005) sample, which was more restrictive and consisted of all 6.7-GHz class II methanol masers with associated 95-GHz emission (a lower detection limit of $\sim 4.5 \mathrm{Jy}$ ) within the Galactic longitude range $l=325^{\circ}-335^{\circ}, b= \pm 0.53^{\circ}$. This means that our sample is statistically complete with a different set of selection biases present than the Voronkov et al. sample. Due to all of our targets (except 333.07-0.45) being previously observed at high-resolution by Voronkov et al. (2014), we can compare all three of the observed class I transitions in our statistically complete maser target sample. Although we have 32 pointing targets total for our data, four of these pointings are associated with two class I sources (two pointings each source), therefore, in general, we will refer to a set of 30 class I sources throughout the discussion, except in cases where we are directly discussing relations between class I emission and the class II sources.

\subsection{Diversity in class I methanol masers}

In comparison to the complex spectra observed from class II methanol masers, class I emission appears to be considerably simpler. The spectra from multiple class I transitions observed from the same masing region often appear as single components with peak emission occurring at a common velocity. This contrasts with the typical spectrum of a class II region, with multiple bright components spread across a large velocity range. However, despite the similarities in appearance of the spectra of class I transitions, our investigation, combined with that of Voronkov et al. (2014), reveals significant differences between the three considered transitions. Our high-resolution observations, when compared to the 36and 44-GHz observations of Voronkov et al. (2014), reveal frequent differences between the physical associations of the $95-\mathrm{GHz}$ maser components with other phenomenon in the star-formation regions. 95-GHz masers are observed tracing ordered structures, both independently or coinciding with other class I transitions. We observe a stronger preference towards co-location with the class II maser position in the $95-\mathrm{GHz}$ masers compared with the $36-$ or $44-\mathrm{GHz}$ components. This observation is particularly interesting due to the shared optimal conditions for masing between the 95- and 44-GHz components (McEwen, Pihlström \& Sjouwerman 2014; Leurini, Menten \& Walmsley 2016). In addition, we see significant offsets in LSR velocity between matched components from the two $A^{+}$. type transitions (44-GHz blue-shifted compared to $95 \mathrm{GHz}$ ), which is not observed between the matched $95-$ and $36-\mathrm{GHz}$ components. This diversity between transitions will be discussed further in the following sections.

\subsection{General associations}

The angular resolution of our observations allows us to determine the association of class I methanol masers and other phenomena observed in star-formation regions. The high angular resolution of the maser data combined with the Spitzer infrared images enables us to investigate association rates between the class I emission and EGOs and other 4.5- $\mu \mathrm{m}$ excess regions (Cyganowski et al. 2008). These regions of enhanced 4.5- $\mu \mathrm{m}$ emission can result from shocked gas (De Buizer \& Vacca 2010), therefore, we expect to see high association rates with class I methanol masers. Voronkov et al. (2014) reported association rates of $\sim 54$ and $\sim 82$ per cent between class I emission and EGOs or any 4.5- $\mu \mathrm{m}$ excess source, respectively, across their entire sample of 64 class I sources. They considered an association to exist if a class I component was within a few arcseconds of the relevant region. Using this criterion for our observations, we found close spatial associations between $95-\mathrm{GHz}$ class I emission and EGOs in 19 out of 30 sources ( 63 per cent). In 4 out of our 30 sources (G326.48+0.70, G328.24-0.55, G332.30-0.09, and G333.03-0.06), 95-GHz class I emission is associated with $4.5-\mu \mathrm{m}$ excess sources that were not determined to be EGOs by Cyganowski et al. (2008), two of these (G326.48+0.70 and G332.30-0.09) have associations with EGOs in addition to these other $4.5-\mu \mathrm{m}$ excess regions. Therefore, we see 21 out of 30 sources ( $\sim 70$ per cent) have some sort of 95-GHz maser emission associated with regions of excess $4.5-\mu \mathrm{m}$ emission. If we instead consider any class I emission (95-GHz masers combined with 36- and 44-GHz masers reported by Voronkov et al. 2014), we determine the following association fractions: 21 out of 30 target sources $(\sim 70$ per cent) contain class 
I masers spatially associated with EGOs as defined by Cyganowski et al. (2008) and 24 out of 30 sources ( 80 per cent) have class I masers spatially associated with any $4.5-\mu \mathrm{m}$ excess source. Therefore, we can conclude that in the majority of cases where both class I and class II methanol maser emission is observed, there is some component of class I maser emission is associated with a likely outflow candidate. Additionally, when comparing the association rates between EGOs and the class I transitions, we see the 95-GHz maser components have an increased association rate with confirmed EGOs compared to the other two class I transitions combined (Cyganowski et al. 2008; Voronkov et al. 2014). A review of single dish surveys of class I maser sources identified that approximately 50 per cent of class I maser sources have emission (44 or 95-GHz) within an arcminute of an outflow source (EGO or otherwise) (Chen, Ellingsen \& Shen 2009), and subsequent searches for class I methanol masers targeted towards EGOs have achieved high detection rates (Chen et al. 2011). Our association rate between outflows and class I emission is significantly higher than this (80 per cent when considering any class I emission in our sources) and unlike the single-dish data, our high-resolution data allow us to directly confirm the presence of class I maser components within these outflow sources.

There are 57 reported EGOs within the Galactic longitude range of our statistically complete sample (Cyganowski et al. 2008). 22 ( $\sim 39$ per cent) of these EGOs have $6.7-\mathrm{GHz}$ class II methanol maser associations and 14 ( $\sim 25$ per cent) have class I methanol maser detections in one or more of the discussed class I transitions (Cyganowski et al. 2008; Voronkov et al. 2014). Indicating that EGOs with associated 6.7-GHz methanol masers make good targets for observing class I methanol masers.

Twenty of the $6.7-\mathrm{GHz}$ class II methanol masers that were observed here have associated $12.2-\mathrm{GHz}$ class II methanol masers (Breen et al. 2012a,b). When considering our statistically complete sample 12 of 21 targets ( $\sim 57$ per cent) are detected in the $12.2-\mathrm{GHz}$ transition. Including all $6.7-\mathrm{GHz}$ masers within the statistically complete sample, our targets were drawn from (all 6.7-GHz masers, regardless of presence of $95-\mathrm{GHz}$ emission) we see an association rate between the two class II transitions (6.7 and $12.2 \mathrm{GHz}$ ) of 60 per cent. Comparing these two rates, we see that $6.7-\mathrm{GHz}$ masers also displaying emission at $95-\mathrm{GHz}$ do not appear to have a significantly different association rate with 12.2$\mathrm{GHz}$ class II masers. Sources with emission present from $12.2-\mathrm{GHz}$ class II methanol masers tend to be at a later evolutionary stage than those without (Breen et al. 2010, 2011). This indicates that 95$\mathrm{GHz}$ class I emission does not appear to favour these more evolved sources.

\subsection{Continuum association}

In addition to the observations of $95-\mathrm{GHz}$ maser emission, we also searched for 3.5-mm continuum in each of our targets. We detected $3.5-\mathrm{mm}$ continuum emission in 14 of our 32 pointings, with two of these pointings corresponding to the one class I methanol maser source. Therefore, 3.5-mm continuum emission is observed in 13 of the 30 ( $\sim 43$ per cent) class I methanol maser sources (typical $3 \sigma$ upper limit is $\sim 7 \mathrm{mJy}$ for non-detections). 7 of our 30 class I sources have previously reported cm-wavelength continuum emission (18-cm emission for all except G328.24-0.55, which has a $3-\mathrm{cm}$ continuum detection) and we detect $3.5-\mathrm{mm}$ continuum in all but one (G333.47-0.16). Voronkov et al. (2014) report an association rate of $\sim 24$ per cent between their class I maser sources and cm-wavelength continuum emission. This is significantly lower than the association rate we observe between the $95-\mathrm{GHz}$ and mmwavelength continuum sources. However, it should be noted that their rate was reported as a lower limit due to the inhomogeneous nature of their continuum data with respect to their sample of sources. It is likely that mm-wavelength continuum emission, such as the observed 3.5-mm emission results from thermal dust, in contrast to $\mathrm{cm}$-wavelength continuum emission which is mostly free-free emission from $\mathrm{H}$ II regions. This indicates that class I sources displaying $\mathrm{mm}$ - and not $\mathrm{cm}$-wavelength continuum emission may be at an earlier evolutionary phase.

In all of these sources we observe $95-\mathrm{GHz}$ masers closely colocated (to within a few arcseconds) with the continuum emission. Additionally, in all sources where continuum emission is observed, at least some component of it was observed within several arcseconds of the class II pointing target. Considering both $\mathrm{cm}$ - and mm-wavelength continuum data for our sources, we obtain an association rate between class II masers and radio continuum emission ( $\sim 47$ per cent) consistent with those previously reported (Phillips et al. 1998; Walsh et al. 1998).

\subsection{ATLASGAL association}

The ATLASGAL survey mapped continuum emission from cold, dense dust and gas throughout the Galactic Plane. All class I methanol maser sources we have observed are within the bounds of the ATLASGAL survey. All of our class I maser regions are closely associated (within 30 arcsec of peak emission) with ATLASGAL sources with the $95-\mathrm{GHz}$ maser spots appearing to be slightly offset from the central peak of the dust emission, which is consistent with the class I masers being associated with gas outflows. We observe a positive linear correlation between the dust mass of these ATLASGAL sources, and the luminosity of the associated $6.7-\mathrm{GHz}$ class II masers with a correlation coefficient $(r)$ of 0.85 .

In the majority of sources that we observe strong $3.5-\mathrm{mm}$ continuum emission, we observe the location of the ATLASGAL point source coinciding with the position of this continuum emission. Additionally, we consider whether sources where $3.5-\mathrm{mm}$ continuum emission or IR sources are associated with the class I maser emission affects the dust masses of the ATLASGAL sources. This was done by using the ANOVA statistical function to determine the significance of the various categorical variables (e.g. presence of EGO, 8- $\mu \mathrm{m}$ IR source, etc.) in predicting ATLASGAL dust mass. The ANOVA function is robust to the normality assumption of the continuous variable (ATLASGAL dust mass) allowing us to be reasonably confident in the output despite the skewed distribution of the dust masses. The presence of continuum emission and 4.5- $\mu \mathrm{m}$ excess sources (whether EGO or other) do not correspond to distributions of ATLASGAL masses with statistically different means. Conversely, association with 8- $\mu \mathrm{m}$ emission does result in a statistically significant difference in the mean, with sources displaying 8- $\mu \mathrm{m}$ emission generally displaying lower dust masses.

In targets where no IR source was observed at the location of the class II maser, the ATLASGAL source likely pinpoints the location of the young star driving the methanol emission. However, in the eight sources where this is the case, we do not observe a close coincidence between the peak of the ATLASGAL emission and the location of the class II masers. We also do not observe any statistically significant difference between the luminosity of class II masers in sources where no IR source is identified versus those with IR sources detected. 


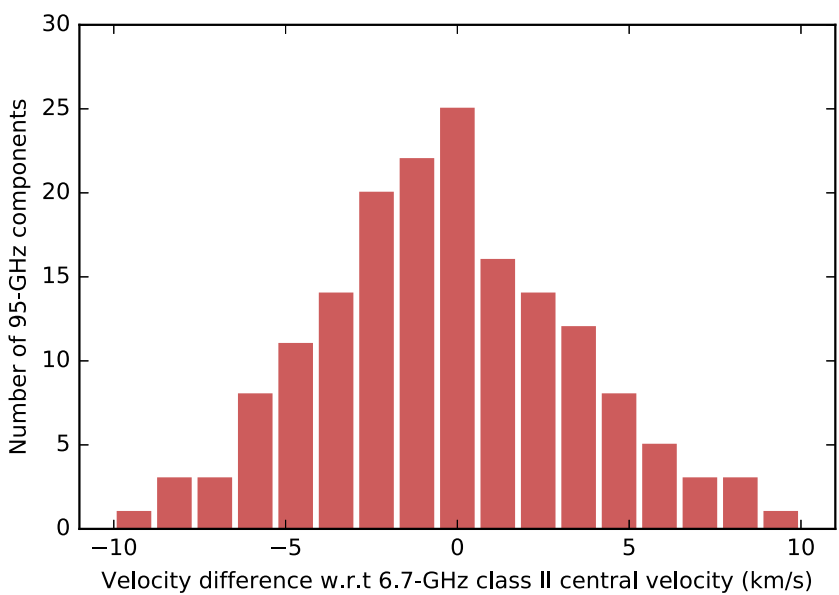

Figure 3. The histogram representing the distribution of velocity separations between the $95-\mathrm{GHz}$ class I masers and the central velocity range value of the 6.7-GHz class II target sources.

\subsection{Velocity separation}

Voronkov et al. (2014) compared relative velocities of the 36-/44$\mathrm{GHz}$ class I methanol masers with the quiescent gas in the same region. Their results were consistent with the expected result that class I methanol masers have a closely related radial velocity to the systemic velocity. We follow up this analysis with a similar comparison using the detected $95-\mathrm{GHz}$ masers. In the Voronkov et al. (2014) study, the velocity of the quiescent gas was approximated by the central velocity value of $6.7-\mathrm{GHz}$ class II methanol maser velocity range. As the pointing targets for our observations are $6.7-\mathrm{GHz}$ class II masers, we will use this same convention in our analysis. Creating a histogram (Fig. 3) of relative velocities, we see that the distribution can be reasonably approximated by a normal distribution with a mean of $-0.33 \pm 0.28 \mathrm{~km} \mathrm{~s}^{-1}$ and a standard deviation of $3.62 \pm 0.20 \mathrm{~km} \mathrm{~s}^{-1}$. Qualitatively the histogram for the $95-\mathrm{GHz}$ masers appears to have a minor blueshift asymmetry, similar to the $36-$ and $44-\mathrm{GHz}$ transitions, however, this shift cannot be considered statistically significant in our data (tested with a one-sample $T$-Test). The ratio of negative to positive relative velocities is 1.34 ; this is statistically significant from unity, however, it requires a simplification of the problem to a binomial distribution and is thus not a rigorous test. Follow-up observations at higher spectral resolution are required in order to conclude whether this is a real result. Unlike the 36- and $44-\mathrm{GHz}$ transitions, we observed no components with extreme relative velocities, with all relative velocities falling within a range of approximately -10 to $10 \mathrm{~km} \mathrm{~s}^{-1}$. The $3 \sigma$ range of our results is very similar to that observed from the other class I transitions presented by Voronkov et al. (2014). Due to the nature of our observing (covering a fixed range of -100 to $0 \mathrm{~km} \mathrm{~s}^{-1}$ ), some sources such as G333.16-0.10, with velocities close to the edge of this range $\left(\sim-92 \mathrm{~km} \mathrm{~s}^{-1}\right)$, will not be sensitive to high-velocity blue-shifted components. This same limitation is not an issue at the opposite end of our velocity range, as we do not observe any sources with systemic velocities higher than $-37 \mathrm{~km} \mathrm{~s}^{-1}$.

\subsection{Comparison with 36-/44-GHz class I masers}

Voronkov et al. (2014) presented a detailed comparison between spatially associated 36- and 44-GHz methanol maser components.
Combining these data with our observations allows for an analysis of the relationship between all three class I methanol maser transitions within our sample. In order to appropriately compare emission from each transition, we need to determine which maser components display emission in more than one transition. This was achieved by matching components between transitions that were coassociated both spatially and with respect to LSR velocity. 95-GHz components were matched with components from the other class I transitions if they are co-located within 1.5 arcsec and separated by $1 \mathrm{~km} \mathrm{~s}^{-1}$ or less in LSR velocity. It is worth noting that while the Voronkov et al. observations of the two different class I transitions occurred quasi-simultaneously, our observations were made $3 \mathrm{yr}$ prior. Therefore, any comparison between matched $95-\mathrm{GHz}$ masers and 36-/44-GHz masers will have inherently more uncertainty than comparison between matched 36- and $44-\mathrm{GHz}$ transitions as variability within class I methanol maser sources is not currently well understood or studied. The 36-/44-GHz masers were grouped using the same method outlined by Voronkov et al., where components from the same transition that were co-located within $3 \sigma$ (in fit uncertainty) of both position and velocity were considered a single component for the purpose of analysis. Across all $17295-\mathrm{GHz}$ components, we find matches with $36-\mathrm{GHz}$ components in 51 cases ( $\sim 30$ per cent) and matches with $44-\mathrm{GHz}$ components in $85(\sim 49$ per cent). 97 out of the 172 ( $\sim 56$ per cent) total components had a match in either of the other class I methanol maser transitions, with 39 components ( $\sim 22$ per cent) displaying emission in all three transitions. Note that the LSR velocity of $36-\mathrm{GHz}$ masers was adjusted in accordance with the rest-frequency corrections described by Voronkov et al. (2014) $\left(-0.215 \mathrm{~km} \mathrm{~s}^{-1}\right)$ before being matched with the 95-GHz masers.

When considering 95-/44-GHz pairs, we find that 57 of 85 cases ( $\sim 67$ per cent) have higher flux density in the $44-\mathrm{GHz}$ transition (left-hand plot, Fig. 5). In the 95-/36-GHz matched pairs, we find that the $95-\mathrm{GHz}$ transition is brighter in 39 out of 51 ( $\sim 76$ per cent) cases (right-hand plot, Fig. 5). The relationship between the 95and $44-\mathrm{GHz}$ maser pairs is expected due to both of these transitions belonging to the same transition series (both are $\mathrm{J}_{0}-(\mathrm{J}-1)_{1} \mathrm{~A}^{+}$ transitions with consecutive $\mathrm{J}$ numbers).

Flux density scatter plots were created between the matched components of each pair of transitions (Fig. 6). Only 36- and 44-GHz maser components from within our source sample were considered, therefore, the comparison between the paired 36- and 44-GHz masers here is a smaller sample size than that in Voronkov et al. (2014). A first-order polynomial line of best fit was determined for each of these plots. Considering the flux density scatter plot of 95and 44-GHz matched components (plot (a) of Fig. 6), the linear fit had a slope of $0.35 \pm 0.10$ (and intercept value of $2.1 \pm 0.5$ ) with a modest correlation coefficient $(r)$ of 0.78 . This relationship is similar to the $3: 1$ ratio between $44-$ and $95-\mathrm{GHz}$ flux density reported by Val'tts et al. (2000). We find reasonable coefficients of correlation ( $r=0.78$ for $95-/ 36-\mathrm{GHz}$ and $95-/ 44-\mathrm{GHz}$ and $r=0.79$ for $44-/ 36-\mathrm{GHz})$ in the linear fits of all three of our transition pairs. It is important to note that these comparisons were made using the flux density peak values for each transition. This introduces velocity resolutions effects when comparing our $95-\mathrm{GHz}$ data to the 36- and 44-GHz values, as the velocity resolution of our observations are approximately a factor of 8 coarser. The observed relationship between frequency of association and flux density is consistent with the expected relationships between these transitions (Cragg et al. 1992; McEwen et al. 2014; Leurini et al. 2016).

When considering the velocity separation between matched class I transitions, we observe a red-shift between the $95-\mathrm{GHz}$ 


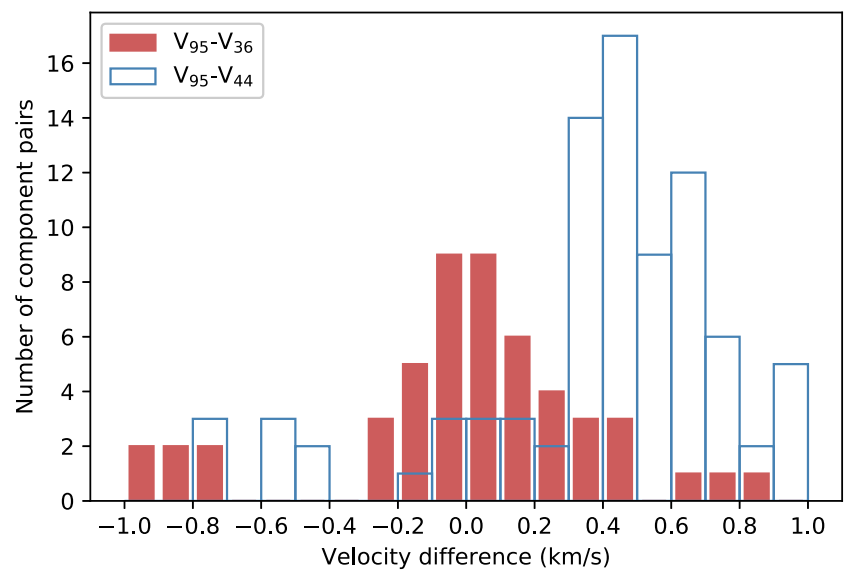

Figure 4. Histograms of velocity offset between matched $95-$ and $36-\mathrm{GHz}$ masers (red filled) and 95- and 44-GHz masers (blue outline).

components with respect to their matched 44-GHz components in 73 out of 85 ( $~ 86$ per cent) pairs with a mean red-shift of $0.385 \mathrm{~km} \mathrm{~s}^{-1}$ over all pairs (see Fig. 4). The velocity separations between the $95-$ and $36-\mathrm{GHz}$ matched pairs has a mean of $0.005 \mathrm{~km} \mathrm{~s}^{-1}$. The distribution of the velocity separation between both pairs of transitions is approximately Gaussian.

It is interesting to consider the number of regions where $95-\mathrm{GHz}$ masers are detected which also contain emission from either, or both, of the other two class I transitions. To do this, we considered the original spatial association of maser components represented by the letter symbols in our images (originally defined in Voronkov et al. 2014). In most cases these locations have a maximum spread of approximately 4 arcsec, which is close enough to assume colocation in the same general environment. In a few cases $95-\mathrm{GHz}$ masers were seen to be offset from an alphabetized location; in these situations they were considered their own region. This resulted in 64 groupings of $95-\mathrm{GHz}$ class I masers over the 30 class I sources. In 52 of the 64 ( $~ 81$ per cent) clusters of 95-GHz masers, both $36-$ and $44-\mathrm{GHz}$ masers are also present. When considering cases where only $36-$ or $44-\mathrm{GHz}$ are co-located with the $95-\mathrm{GHz}$ masers, this reduces down to $\sim 1.5$ per cent ( 1 region) and $\sim 14$ per cent ( 9 regions), respectively, with the remaining two regions ( $\sim 3$ per cent; regions in G 328.25-0.53 and G 329.07-0.31) only containing $95-\mathrm{GHz}$ class I masers. Therefore, the vast majority of regions where $95-\mathrm{GHz}$ masers are detected have $36-$ and $44-\mathrm{GHz}$

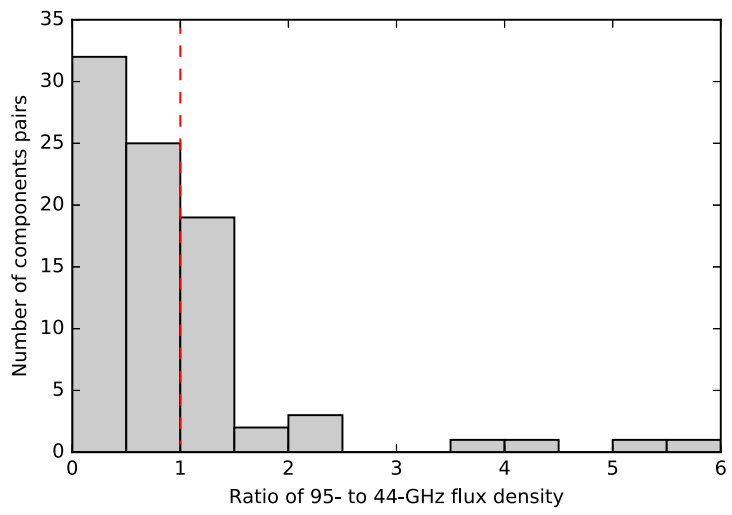

masers in relatively close proximity. Different environmental conditions favour different class I methanol transitions (Cragg et al. 1992; McEwen et al. 2014; Leurini et al. 2016). For the three transitions considered here, the $44-\mathrm{GHz}$ and $95-\mathrm{GHz}$ emission are related and share similar optimal masing conditions, whereas the $36-\mathrm{GHz}$ emission prefers denser environments. All three transitions favour temperatures $\geq 50 \mathrm{~K}$, however, the optimal density range (of molecular hydrogen) for the two $A^{+}$type transitions is between $10^{4}$ and $10^{6} \mathrm{~cm}^{-3}$, compared with $10^{5}-10^{7} \mathrm{~cm}^{-3}$ for the $36-\mathrm{GHz}$ transition (McEwen et al. 2014). Masers from the 36- and 44-GHz transitions can be observed co-spatially due to overlapping optimal conditions for masing (Voronkov et al. 2010, 2014; Pihlström, Sjouwerman \& Fish 2011; McEwen, Pihlström \& Sjouwerman 2014). Therefore, as the $95-\mathrm{GHz}$ masers have similar optimal conditions as the $44-\mathrm{GHz}$ transition, this explains why we tend to observe spatial associations between all three transitions in regions where $95-\mathrm{GHz}$ components are located (which are in general optimal for 44-/95-GHz masing, allowing 95-GHz to be observable).

\subsection{YSO range of influence}

Due to the nature of their pumping mechanism, 6.7-GHz class II methanol masers are located close to high-mass YSOs. This allows the positions of these class II masers to be used as an accurate indicator of the location of a YSO. As all of our target sources were 6.7-GHz class II masers, we can determine linear offsets between all our detected maser components and nearby YSOs. Kinematic distance estimates to each source were taken from Green \& McClureGriffiths (2011) or computed using similar methodology (see Table 1). Similar analysis for the 36- and 44-GHz class I emission was conducted by Voronkov et al. (2014) and concluded that $1 \mathrm{pc}$ is a good estimate of the range of influence of YSOs in producing class I methanol masers. In the case of our $95-\mathrm{GHz}$ observations, we have a much smaller primary beam which does not allow us to accurately determine whether the $1 \mathrm{pc}$ estimate applies to the 95-GHz masers we observed. Instead, with our data we considered a range out to $0.46 \mathrm{pc}$, this range was determined from the mean linear radius of the primary beam over all of our sources. Considering only the subset of $95-\mathrm{GHz}$ components within this range limit allows for a sample with reduced bias towards detections close to the pointing centre. This reduced sample can be compared against the 36- and 44-GHz maser components, which satisfy the same criteria, in order to investigate any positional preference of $95-\mathrm{GHz}$ masers towards YSOs relative to the 36- and 44-GHz masers.

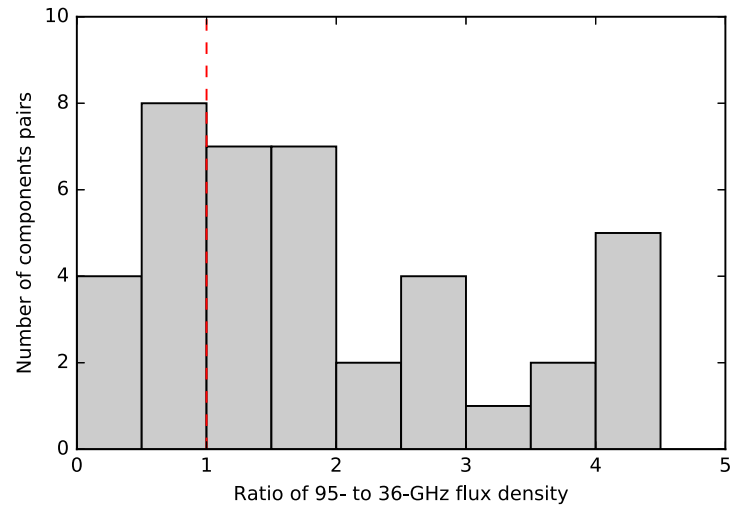

Figure 5. Distribution of the flux density ratio of 95 - to $44-\mathrm{GHz}$ masers in matched pairs (left) and 95 - to $36-\mathrm{GHz}$ pairs (right). There are 85 and 51 matched pairs in the 95-/44-GHz and 95-/36-GHz distributions, respectively. Vertical dashed line separates the pairings where 95-GHz component is brighter than the other paired class I methanol maser transition. 


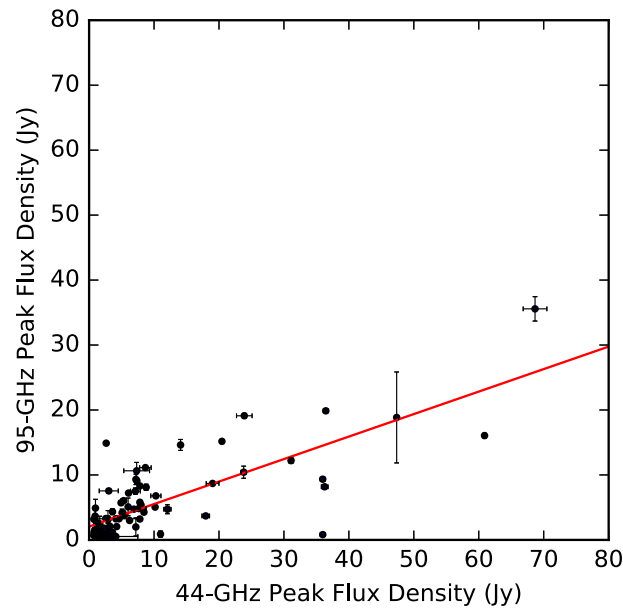

(a)

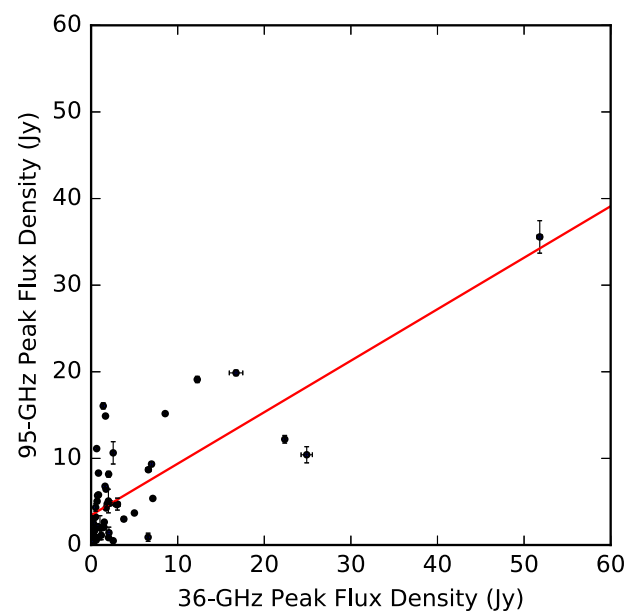

(b)

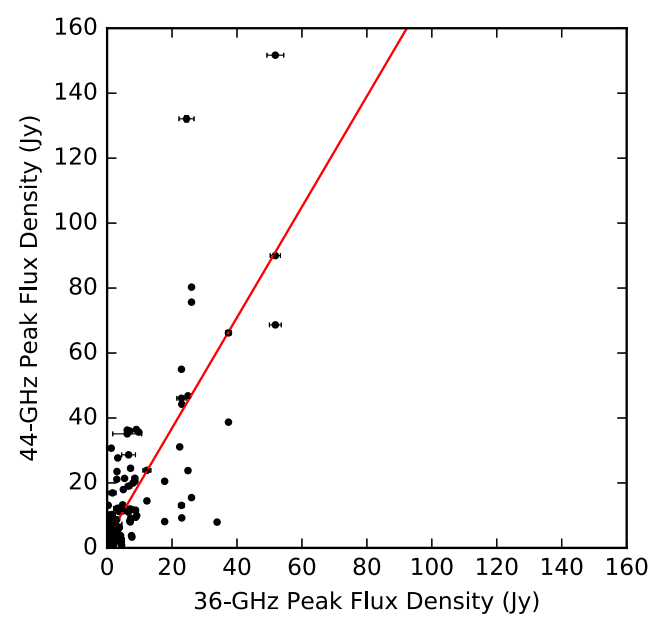

(c)

Figure 6. Scatter plots of peak flux density between matched class I components, 95- and 44-GHz (a), 95- and 36-GHz (b) and 44- and 36-GHz (c). Line in each plot is a first-order polynomial best fit: $y=(0.35 \pm 0.10) x-(2.1 \pm 0.5)(a), y=(0.59 \pm 0.17) x-(3.4 \pm 0.7)(b)$, $y=(1.70 \pm 0.49) x-(2.8 \pm 1.3)(\mathrm{c})$.

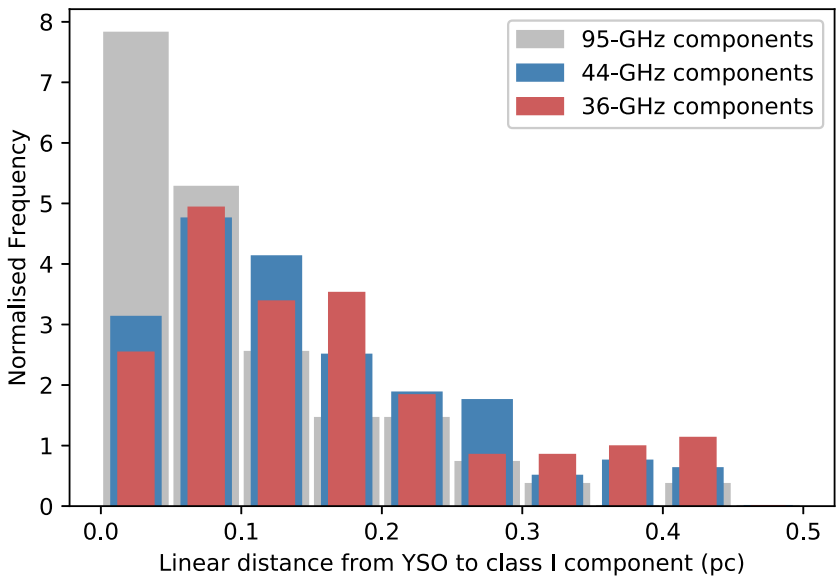

Figure 7. The normalized histogram of class I components linear separation from the $6.7-\mathrm{GHz}$ (indicative of the position of the YSO). This is the reduced sample, of only components within $0.46 \mathrm{pc}$ of the excitation source (see Section 4.7). The histogram for each frequency has been normalized so that the $y$-axis is the value of the probability density function for each bin, ensuring across the entire sampled range this integrates to 1 . This allows comparison between the three samples of class I masers, despite sample sizes not being equal.

Five of our 32 target sources were excluded from this analysis: one due to a missed pointing (G333.56-0.02) and another four due to multiple close $6.7-\mathrm{GHz}$ class II masers present in the field (G332.30-0.09, G 333.13-0.44, G 333.13-0.56, and G333.23-0.06). Sources with multiple 6.7-GHz masers are excluded as we are unable to determine which class II source the class I masers are associated with. In the remaining 27 sources, we determined linear offsets for 138 components, with 127 of these falling within the range of $0.46 \mathrm{pc}$ from a YSO. Within this subset of 127 components, we observe 81 ( $\sim 59$ per cent) with projected distances less than $0.1 \mathrm{pc}$ and $107(\sim 78$ per cent) less than $0.2 \mathrm{pc}$. Therefore, we observe more than an $\sim 68$ per cent drop in the number of components when comparing those from between 0.1 and $0.2 \mathrm{pc}$ and those within $0.1 \mathrm{pc}$ of the YSO. Considering a 2-dimensional density determined as the number of components per projected area, the density of the region between 0.1 and $0.2 \mathrm{pc}$ from the YSO is $\sim 89$ per cent lower than that of the inner region. When considering components from all three class I transitions within this same $0.46 \mathrm{pc}$ range, we must exclude three additional sources (G 329.03-0.20, G329.03-0.19, and G333.12-0.43) as these have multiple 6.7-GHz class II masers within the FWHM of the 36- and 44$\mathrm{GHz}$ primary beam. In this reduced sample, we observe $\sim 65$ per cent of 95-GHz class I masers within $0.1 \mathrm{pc}$ of the YSO, compared to the $\sim 37$ and $\sim 39$ per cent observed for the 36- and $44-\mathrm{GHz}$ transitions, respectively (see Fig. 7). Despite considering a reduced sample to alleviate bias towards $95-\mathrm{GHz}$ maser components nearby to YSOs, this sample is only appropriate for comparing between the three transitions and are not indicative of the true distribution of 95-GHz maser emission in these sources. In order to accurately determine the distribution of $95-\mathrm{GHz}$ maser spots with respect to the YSO, multiple pointings of each source would need to be made or an instrument with a much larger primary beam utilized.

The result that $95-\mathrm{GHz}$ masers appear to be found closer to the exciting source than the other two transitions would seem to indicate that the $95-\mathrm{GHz}$ transition prefers a comparatively higher energy environment. However, the both 44- and 95-GHz share similar conditions for ideal population inversion (Cragg et al. 1992; 
McEwen et al. 2014), and we do not see as strong a preference for higher energy environments in the former. There is one observational factor that may be responsible for the perceived difference between these two related transitions. The $44-\mathrm{GHz}$ maser transition is generally the stronger of the two transitions, so if the $95-\mathrm{GHz}$ needs a higher energy environment for observable masing to occur, we may see a steeper decline in detected components when in lower energy areas, due to the sensitivity bias of our observations. This will have the effect of making $95-\mathrm{GHz}$ masers seem proportionally closer to the excitation source than the $44-\mathrm{GHz}$ masers. Additionally, we see no correlation between the flux density of a component (of any class I transition) and its linear separation from the associated YSO, which is consistent with the findings of Voronkov et al. (2014).

\subsection{Class I-Class II association at high resolution}

While the vast majority of class I methanol maser components are generally located within $1 \mathrm{pc}$ of a YSO (Voronkov et al. 2014), typically, class I emission is not observed coincident with class II methanol maser features. Kurtz et al. (2004) observed examples of spatial association between $44-\mathrm{GHz}$ class I and $6.7-\mathrm{GHz}$ class II masers, and suggested that the mutual exclusivity between the two classes may not be as strong as previously supposed. In Section 4.7 we observe the majority of $95-\mathrm{GHz}$ emission nearby $(<0.2 \mathrm{pc})$ to the YSO (marked by the location of the $6.7-\mathrm{GHz}$ masers) and Fig. 3 shows the distribution of velocities for our components is approximately Gaussian about the class II central velocity. Therefore, we should observe some $95-\mathrm{GHz}$ maser components, both spatially associated and within the velocity range of the $6.7-\mathrm{GHz}$ class II masers. Observation of components matching these two conditions does not conclusively mean that masers from the two classes are associated with the same environment and could instead result simply from chance alignment.

We include in Table 1 the linear offset between each class II maser and the closest $95-\mathrm{GHz}$ component coincident with the class II maser velocity range. In 8 (of 32) class II sources we observe class I maser emission both: closer than 1 arcsec in position and within the velocity range of the 6.7-GHz masers (Caswell et al. 2011). The median angular separation and projected linear separation of all 95$\mathrm{GHz}$ components satisfying the aforementioned criteria is 0.71 arcsec and 0.013 pc, respectively. G 328.25-0.53 and G 333.03-0.06 host the two most accurately positioned components within this subset, with $3 \sigma$ angular errors in their fitted positions of $<0.2$ arc$\mathrm{sec}$. The line-of-site distance to these sources is less than $3 \mathrm{kpc}$ (see Table 1), therefore, these small angular offsets correspond to projected linear distances of less than $0.01 \mathrm{pc}$. In contrast to the $95-\mathrm{GHz}$ masers, similar spatial alignment between class I maser components and $6.7-\mathrm{GHz}$ masers was only observed in three sources from our sample, for both the 36- and 44-GHz transitions. Such a considerable difference between the $95-$ and $44-\mathrm{GHz}$ masers is interesting due to their shared optimal environment conditions (Cragg et al. 1992; McEwen et al. 2014), however, it is worth noting that we are only considering 44-GHz emission from sources we have observed at $95 \mathrm{GHz}$.

From our observations it is not possible to definitively determine whether these components are anything other than aligned with the line of sight to the class II source. In order to properly investigate coincidence between class I and class II masers, high-resolution astrometry must be performed. However, these close angular offsets combined by the general trend that $95-\mathrm{GHz}$ masers appear preferentially towards the driving source (discussed in Section 4.7) may indicate that masers from this transition are more strongly inverted when close to a background continuum source.

\section{CONCLUSIONS}

We present $95-\mathrm{GHz}$ imaging results from 32 pointings of a statistically complete sample of $6.7-\mathrm{GHz}$ class II methanol masers. We detected $95-\mathrm{GHz}$ maser emission in all pointings and across all 32 pointings we detected a total of $17295-\mathrm{GHz}$ class I methanol components. Due to two cases with dual pointings, these 32 pointings represent coverage of 30 individual class I methanol maser sources. In many cases we observe similar associations between these $95-\mathrm{GHz}$ maser components and ordered structures, as is seen in 36-/44-GHz components in the same sources (Voronkov et al. 2014). We observe associations between $95-\mathrm{GHz}$ components and 4.5 - $\mu \mathrm{m}$ excess sources in 21 out of 30 sources ( 70 per cent) or 24 out of 30 sources ( 80 per cent) when considering all class I methanol emission (inclusion of 36-/44-GHz components). Additionally, in 19 of 30 sources ( $\sim 63$ per cent) we observe associations with $8.0-\mu \mathrm{m}$ emission, increasing to 22 of 30 sources ( $\sim 73$ per cent) with the inclusion of all class I methanol data for these sources. 3.5mm continuum sources were detected in 13 of our 30 sources $(\sim 43$ per cent) and in all of these sources we see co-location (within a few arcseconds) between the continuum emission, the $95-\mathrm{GHz}$ class I masers, and the 6.7-GHz class II maser.

When considering our statistically complete sample (all 6.7-GHz class II sources with observed 95-GHz maser emission), we observe a 57 per cent association rate with $12.2-\mathrm{GHz}$ class II masers). This is not significantly different from the 60 per cent association rate observed between the two class II transitions in the larger complete sample that our targets were drawn from. As 12.2-GHz masers generally appear at later evolutionary stages, the $95-\mathrm{GHz}$ masers do not seem to prefer more evolved sources.

We observe component matches between the $44-\mathrm{GHz}$ and 95-GHz transitions in 85 ( $\sim 49$ per cent $)$ of our detected $95-\mathrm{GHz}$ components and matches between 36- and $95-\mathrm{GHz}$ transitions in 51 ( $\sim 30$ per cent) $95-\mathrm{GHz}$ components. Only 39 of our 172 observed 95-GHz components have matching components from all three transitions. These matches were made by cross-matching every $95-\mathrm{GHz}$ maser with its nearest 36- and 44-GHz masers, then filtering these matches with a limit of 1.5 arcsec of angular separation and $1 \mathrm{~km} \mathrm{~s}^{-1}$ of LSR velocity separation. Additionally when considering broader environmental associations between the two transitions, we find that in $\sim 81$ per cent of regions containing $95-\mathrm{GHz}$ components, 36 - and 44-GHz components are also observed. Indicating that the vast majority of environments with suitable conditions for $95-\mathrm{GHz}$ masing are also suitable for $36-$ and $44-\mathrm{GHz}$ masing. When comparing the flux densities between matched 44- and 95-GHz components, we observe a similar relationship to the 3:1 ratio reported by Val'tts et al. (2000). Indicating that this relationship holds when considering high-resolution data of both transitions where individual maser components can be matched together.

When comparing the velocities of matched class I components, we observe a red-shift of $0.385 \mathrm{~km} \mathrm{~s}^{-1}$ on average between 95- and 44-GHz components. No significant shift is observed between the 95- and 36-GHz matched components.

When considering the offset from the YSOs (position defined as location of the 6.7-GHz masers), 95-GHz maser spots appear to be preferentially closer than the other considered class I transitions. This could be explained by the 95-GHz methanol transition being more strongly inverted when closer to a background continuum source. Additionally, we observe some $95-\mathrm{GHz}$ components at very 
small linear offsets from class II 6.7-GHz masers, however, highresolution astrometry is required to determine whether any real association exists.

The distribution of velocity offsets between $95-\mathrm{GHz}$ components and the systematic source velocity (defined by the central velocity value of the $6.7-\mathrm{GHz}$ class II emission in each source) has a mean of $-0.33 \pm 0.28 \mathrm{~km} \mathrm{~s}^{-1}$. We observe no high-velocity features in any of our sources at $95 \mathrm{GHz}$.

Similar to the complementary nature of the $36-$ and $44-\mathrm{GHz}$ components observed in our target sources (Voronkov et al. 2014), there are many cases where the $95-\mathrm{GHz}$ components trace the same morphological structures in a particular source. In several of these cases, the $95-\mathrm{GHz}$ components extend along paths not covered by 36- or 44-GHz components. Therefore, observations in all three transitions can be useful in determining the exact structure of particular morphological features. Our investigation rejects the notion that the class I maser transitions of the methanol species are simpler than class II, with diversity in relationships and associations being observed between closely related transitions $(95-\mathrm{GHz}$ and 44-GHz $A$-type).

\section{ACKNOWLEDGEMENTS}

We thank the anonymous referee for useful suggestions which helped to improve this paper. The ATCA is part of the Australia Telescope, which is funded by the Commonwealth of Australia for operation as a National Facility managed by CSIRO. This research has made use of NASA's Astrophysics Data System Abstract Service. This research has made use of data products from the GLIMPSE survey, which is a legacy science programme of the Spitzer Space Telescope, funded by the National Aeronautics and Space Administration, and the NASA/IPAC Infrared Science Archive, which is operated by the Jet Propulsion Laboratory, California Institute of Technology, under contract with the National Aeronautics and Space Administration. This research also utilized APLPY, an open-source plotting package for PYTHON hosted at http://aplpy.github.com. This research made use of Astropy, a community-developed core PYTHON package for Astronomy (Astropy Collaboration 2013).

\section{REFERENCES}

Astropy Collaboration, 2013, A\&A, 558, A33

Breen S. L., Ellingsen S. P., Caswell J. L., Lewis B. E., 2010, MNRAS, 401, 2219

Breen S. L., Ellingsen S. P., Caswell J. L., Green J. A., Fuller G. A., Voronkov M. A., Quinn L. J., Avison A., 2011, ApJ, 733, 80

Breen S. L., Ellingsen S. P., Caswell J. L., Green J. A., Voronkov M. A., Fuller G. A., Quinn L. J., Avison A., 2012a, MNRAS, 421, 1703

Breen S., Ellingsen S., Caswell J., Green J., Voronkov M., Fuller G., Quinn L., Avison A., 2012b, MNRAS, 426, 2189

Breen S. L. et al., 2015, MNRAS, 450, 4109

Caswell J. L., 1997, MNRAS, 289, 203

Caswell J. L. et al., 2010, MNRAS, 404, 1029

Caswell J. L. et al., 2011, MNRAS, 417, 1964

Chen X., Ellingsen S. P., Shen Z.-Q., 2009, MNRAS, 396, 1603
Chen X., Ellingsen S. P., Shen Z.-Q., Titmarsh A., Gan C.-G., 2011, ApJS, 196,9

Cragg D. M., Johns K. P., Godfrey P. D., Brown R. D., 1992, MNRAS, 259, 203

Cragg D. M., Sobolev A. M., Godfrey P. D., 2005, MNRAS, 360, 533

Cyganowski C. J. et al., 2008, AJ, 136, 2391

Cyganowski C. J., Brogan C. L., Hunter T. R., Churchwell E., 2009, ApJ, 702,1615

Cyganowski C. J., Brogan C. L., Hunter T. R., Zhang Q., Friesen R. K., Indebetouw R., Chandler C. J., 2012, ApJ, 760, L20

De Buizer J. M., Vacca W. D., 2010, AJ, 140, 196

Ellingsen S. P., 2005, MNRAS, 359, 1498

Ellingsen S. P., Shabala S. S., Kurtz S. E., 2005, MNRAS, 357, 1003

Ellingsen S. P., Sobolev A. M., Cragg D. M., Godfrey P. D., 2012, ApJ, 759, L5

Green J. A., McClure-Griffiths N. M., 2011, MNRAS, 417, 2500

Green J. A. et al., 2010, MNRAS, 409, 913

Green J. A. et al., 2012, MNRAS, 420, 3108

Green J. A. et al., 2017, MNRAS, 469, 1383

Kurtz S., Hofner P., Álvarez C. V., 2004, ApJS, 155, 149

Leurini S., Menten K. M., Walmsley C. M., 2016, A\&A, 592, A31

McEwen B. C., Pihlström Y. M., Sjouwerman L. O., 2014, ApJ, 793, 133

Müller H. S. P., Menten K. M., Mäder H., 2004, A\&A, 428, 1019

Peretto N., Fuller G. A., 2009, A\&A, 505, 405

Phillips C. J., Norris R. P., Ellingsen S. P., McCulloch P. M., 1998, MNRAS, 300, 1131

Pihlström Y. M., Sjouwerman L. O., Fish V. L., 2011, ApJ, 739, L21

Sjouwerman L. O., Murray C. E., Pihlström Y. M., Fish V. L., Araya E. D., 2010, ApJ, 724, L158

Slysh V. I., Kalenskii S. V., Valtts I. E., Otrupcek R., 1994, MNRAS, 268, 464

Sobolev A. M., Cragg D. M., Godfrey P. D., 1997, A\&A, 324, 211

Val'tts I. E., Ellingsen S. P., Slysh V. I., Kalenskii S. V., Otrupcek R., Larionov G. M., 2000, MNRAS, 317, 315

Voronkov M. A., Sobolev A. M., Ellingsen S. P., Ostrovskii A. B., 2005, MNRAS, 362, 995

Voronkov M. A., Brooks K. J., Sobolev A. M., Ellingsen S. P., Ostrovskii A. B., Caswell J. L., 2006, MNRAS, 373, 411

Voronkov M. A., Caswell J. L., Ellingsen S. P., Sobolev A. M., 2010, MNRAS, 405, 2471

Voronkov M. A., Caswell J. L., Ellingsen S. P., Green J. A., Breen S. L., 2014, MNRAS, 439, 2584

Walsh A. J., Burton M. G., Hyland A. R., Robinson G., 1998, MNRAS, 301, 640

\section{SUPPORTING INFORMATION}

Supplementary data are available at MNRAS online.

\section{field_images_pdf.tar.gz spectra.tar.gz}

Please note: Oxford University Press is not responsible for the content or functionality of any supporting materials supplied by the authors. Any queries (other than missing material) should be directed to the corresponding author for the article.

This paper has been typeset from a $\mathrm{T}_{\mathrm{E}} \mathrm{X} / \mathrm{L} \mathrm{T}_{\mathrm{E}} \mathrm{X}$ file prepared by the author. 